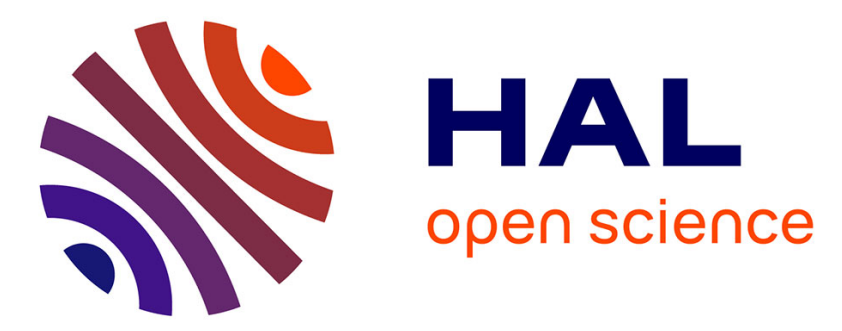

\title{
Convergence of the MAC scheme for the compressible Stokes equations
}

Robert Eymard, Thierry Gallouët, Raphaele Herbin, Jean-Claude Latché

\section{To cite this version:}

Robert Eymard, Thierry Gallouët, Raphaele Herbin, Jean-Claude Latché. Convergence of the MAC scheme for the compressible Stokes equations. SIAM Journal on Numerical Analysis, 2010, 48 (6), pp.2218-2246. 10.1137/090779863 . hal-00542676

\section{HAL Id: hal-00542676 https://hal.science/hal-00542676}

Submitted on 3 Dec 2010

HAL is a multi-disciplinary open access archive for the deposit and dissemination of scientific research documents, whether they are published or not. The documents may come from teaching and research institutions in France or abroad, or from public or private research centers.
L'archive ouverte pluridisciplinaire HAL, est destinée au dépôt et à la diffusion de documents scientifiques de niveau recherche, publiés ou non, émanant des établissements d'enseignement et de recherche français ou étrangers, des laboratoires publics ou privés. 


\title{
CONVERGENCE OF THE MAC SCHEME FOR THE COMPRESSIBLE STOKES EQUATIONS
}

\author{
R. EYMARD *, T. GALLOUËT ${ }^{\dagger}$, R. HERBIN $\ddagger$, AND J.-C. LATCHÉ $\S$
}

September 20, 2010

\begin{abstract}
We prove in this paper the convergence of the Marker and Cell (MAC) scheme for the discretization of the steady state compressible Stokes equations on two or three dimensional Cartesian grids. Existence of a solution to the scheme is proven, followed by estimates on approximate solutions, which yield the convergence of the approximate solutions, up to a subsequence, and in an appropriate sense. We then prove that the limit of the approximate solutions satisfies the mass and momentum balance equations, as well as the equation of state, which is the main difficulty of this study.
\end{abstract}

Key words. Compressible Stokes equations, finite volume methods, MAC scheme

AMS subject classifications. $65 \mathrm{M} 12$

1. Introduction. Since the introduction of the Marker-and-Cell (MAC) scheme [11], it is claimed that this discretization is suitable for both incompressible and compressible flow problems (see $[9,10]$ for the seminal papers, $[2,12,13,14,1,3$, $22,23,24,25,26]$ for subsequent developments and [27] for a review). The use of the MAC scheme in the incompressible case is now standard, and the underlying convergence theory is wellknown. On the contrary, even if some references in the literature report the satisfactory behaviour of this discretization for compressible flow problems, up to our knowledge, no stability nor convergence proof is known in this context. This is the goal of this work, restricting ourselves to the stationary case and to the Stokes problem. The extension to Navier-Stokes equations will be addressed in forthcoming papers. Adapting at the discrete level the arguments of the recent theory of compressible Navier-Stokes equations [17, 6, 20], we prove the existence of a solution to the discrete stationary Stokes equations, and the convergence (up to a subsequence, since, up to now, no uniqueness result is available for the continuous case) of this solution towards a weak solution of the continuous problem as the mesh size goes to 0 . This convergence result seems to be the first one for the compressible Stokes problem in the finite volume context. Convergence results for the same problem can be found in $[7,5,16]$ for the Crouzeix-Raviart element, and, with Navier slip conditions, in [15] for a mixed finite element discretization.

The paper is organized as follows. We first state the continuous problem (Section 2 ), then we present the discrete spaces, norms and operators of interest (Section 3). The scheme is then given (Section 4), and the remainder of the paper is devoted to its analysis: existence and estimates for the discrete solution (Section 5), convergence to a limit satisfying (under a weak form) the differential equations of the continuous problem (Section 6), and finally the equation of state (Section 7).

For the sake of simplicity, we describe the MAC discretization of the compressible Stokes problem in the two-dimensional case, for the square computational domain $\Omega=(0,1)^{2}$. However, the presented material extends to the three-dimensional case

\footnotetext{
${ }^{*}$ Université Paris-Est (eymard@univ-mlv.fr)

$\dagger$ Université de Provence (gallouet@latp.univ-mrs.fr)

†Université de Provence (herbin@latp.univ-mrs.fr)

$\S$ Institut de Radioprotection et de Sûreté Nucléaire (IRSN) (jean-claude.latche@irsn.fr)
} 
and to any bounded domain adapted to the MAC-scheme (that is any finite union of rectangles in $2 \mathrm{D}$ or rectangular parallelipeds in $3 \mathrm{D}$ ); whenever the extension is not straightforward, we give the details of the generalisation.

2. The continuous problem. Let $\Omega$ be an open bounded domain of $\mathbb{R}^{d}$ with $d=2$ or 3 , and let $\gamma \geq 1$. For $\boldsymbol{f} \in \mathrm{L}^{2}(\Omega)^{d}$ and $M>0$, we consider the following problem:

$$
\begin{aligned}
& -\Delta \boldsymbol{u}+\nabla p=\boldsymbol{f} \text { in } \Omega, \quad \boldsymbol{u}=0 \text { on } \partial \Omega, \\
& \operatorname{div}(\rho \boldsymbol{u})=0 \text { in } \Omega, \rho \geq 0 \text { in } \Omega, \int_{\Omega} \rho(x) \mathrm{d} \boldsymbol{x}=M, \\
& p=\rho^{\gamma} \text { in } \Omega .
\end{aligned}
$$

A weak solution of this problem is a function $(\boldsymbol{u}, p, \rho) \in \mathrm{H}_{0}^{1}(\Omega)^{d} \times \mathrm{L}^{2}(\Omega) \times \mathrm{L}^{2 \gamma}(\Omega)$ satisfying:

$$
\begin{aligned}
& \int_{\Omega} \boldsymbol{\nabla} \boldsymbol{u}: \boldsymbol{\nabla} \boldsymbol{v} \mathrm{d} \boldsymbol{x}-\int_{\Omega} p \operatorname{div}(\boldsymbol{v}) \mathrm{d} \boldsymbol{x}=\int_{\Omega} \boldsymbol{f} \cdot \boldsymbol{v} \mathrm{d} \boldsymbol{x} \text { for all } \boldsymbol{v} \in \mathrm{H}_{0}^{1}(\Omega)^{d}, \\
& \int \rho \boldsymbol{u} \cdot \boldsymbol{\nabla} \varphi \mathrm{d} \boldsymbol{x}=0 \text { for all } \varphi \in \mathrm{W}^{1, \infty}(\Omega), \\
& \rho \geq 0 \text { a.e. in } \Omega, \int_{\Omega} \rho \mathrm{d} \boldsymbol{x}=M, p=\rho^{\gamma} \text { a.e. in } \Omega .
\end{aligned}
$$

REMARK 2.1. Note that thanks to the fact that $\boldsymbol{u} \in \mathrm{H}_{0}^{1}(\Omega)^{d}$ and $\rho \in \mathrm{L}^{2}(\Omega)$, one could replace $\varphi \in \mathrm{W}^{1, \infty}(\Omega)$ by $\varphi \in \mathrm{C}_{c}^{\infty}(\Omega)$, see Lemma A.1 in the Appendix. However, this is no longer true if $\rho \in \mathrm{L}^{q}(\Omega)$ for $q<2$ (with $q \geq 6 / 5$ if $d=3$ and $q>1$ if $d=2$ in order for $\rho \boldsymbol{u}$ to belong to $\left.\mathrm{L}^{1}(\Omega)^{d}\right)$.

3. Discrete unknowns and function spaces. For simplicity, we now suppose $\Omega=(0,1)^{2}$ and describe the MAC grid, which we will call $\mathcal{M}$ in the following. Let $N, M \in \mathbb{N}, N, M \geq 2$ and let $\left\{h_{i}^{x}, i=1, \ldots, N\right\}$ and $\left\{h_{j}^{y}, i=j, \ldots, M\right\}$ be two families of positive numbers such that:

$$
\sum_{i=1}^{N} h_{i}^{x}=\sum_{j=1}^{M} h_{j}^{y}=1,
$$

and let us define the step size $h$ of the discretization by:

$$
h=\max \left\{h_{1}^{x}, \ldots, h_{N}^{x}, h_{1}^{y}, \ldots, h_{M}^{y}\right\} .
$$

For the estimates and convergence proof, we need to assume the quasi-uniformity of the mesh, namely that there exists $\eta>0$ such that:

$$
\eta h \leq h_{i}^{x}, h_{j}^{y} \leq h \text { for } 1 \leq i \leq N, 1 \leq j \leq M .
$$

Let $\left(x_{i-\frac{1}{2}}\right)_{1 \leq i \leq N+1}$ and $\left(y_{j-\frac{1}{2}}\right)_{1 \leq j \leq M+1}$ be the families of real numbers defined by:

$$
\begin{aligned}
& x_{\frac{1}{2}}=0, \quad x_{i+\frac{1}{2}}-x_{i-\frac{1}{2}}=h_{i}^{x} \quad\left(\text { so that } x_{N+\frac{1}{2}}=1\right), \\
& y_{\frac{1}{2}}=0, \quad y_{j+\frac{1}{2}}-y_{j-\frac{1}{2}}=h_{j}^{y} \quad\left(\text { so that } y_{M+\frac{1}{2}}=1\right) \text {. }
\end{aligned}
$$




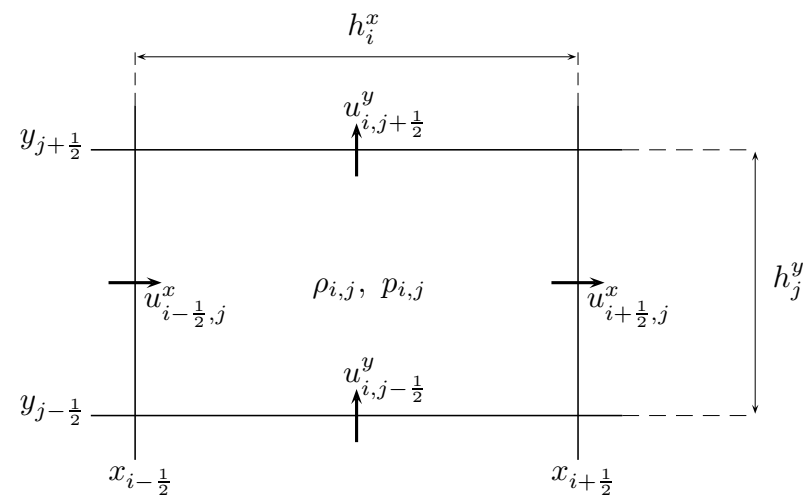

FIG. 3.1. Mesh and unknowns.

We also define $\left(x_{i}\right)_{0 \leq i \leq N+1}$ and $\left(y_{i}\right)_{0 \leq j \leq M+1}$ :

$$
\begin{aligned}
& x_{0}=0, \quad x_{i}=\frac{1}{2}\left(x_{i-\frac{1}{2}}+x_{i+\frac{1}{2}}\right) \text { for } 1 \leq i \leq N, \quad x_{N+1}=1 \text {, } \\
& y_{0}=0, \quad y_{j}=\frac{1}{2}\left(y_{j-\frac{1}{2}}+y_{j+\frac{1}{2}}\right) \text { for } 1 \leq j \leq M, \quad y_{M+1}=1 \text {, }
\end{aligned}
$$

and set:

$$
\begin{array}{ll}
h_{i-\frac{1}{2}}^{x}=\frac{1}{2}\left(h_{i-1}^{x}+h_{i}^{x}\right), & h_{0}^{x}=0, h_{N+1}^{x}=0, \\
h_{j-\frac{1}{2}}^{y}=\frac{1}{2}\left(h_{j-1}^{y}+h_{j}^{y}\right), & h_{0}^{y}=0, h_{M+1}^{y}=0 .
\end{array}
$$

We can now define the following three partitions of $\Omega=(0,1)^{2}$ :

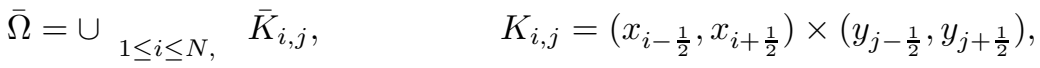

$$
\begin{aligned}
& \bar{\Omega}=\cup_{1 \leq i \leq N+1}, \quad \bar{K}_{i-\frac{1}{2}, j}^{x}, \quad K_{i-\frac{1}{2}, j}^{x}=\left(x_{i-1}, x_{i}\right) \times\left(y_{j-\frac{1}{2}}, y_{j+\frac{1}{2}}\right), \\
& \bar{\Omega}=\cup \underset{\substack{1 \leq i \leq N, 1<j<M+1}}{1<j<\frac{1}{2}}, \quad K_{i, j-\frac{1}{2}}^{y}=\left(x_{i-\frac{1}{2}}, x_{i+\frac{1}{2}}\right) \times\left(y_{j-1}, y_{j}\right) .
\end{aligned}
$$

These three partitions form the "MAC grid"; they are used respectively for the discretization of the pressure and the density, the $x$-component of the velocity and the $y$ component of the velocity. We then define three discrete function spaces, $S \subset \mathrm{L}^{\infty}(\Omega)$, $S^{x} \subset \mathrm{L}^{\infty}(\Omega)$ and $S^{y} \subset \mathrm{L}^{\infty}(\Omega)$, which stand for piecewise constant functions over each of the grid cells $K, K^{x}$ and $K^{y}$ respectively, and the discrete unknowns for the problem are such that the (discrete) pressure $p$ belongs to $S$, the density $\rho$ belongs to $S$, the $x$-component of the velocity $u^{x}$ belongs to $S^{x}$ and the $y$-component of the velocity $u^{y}$ belongs to $S^{y}$. The subdomains $K, K^{x}$ and $K^{y}$ will be referred to hereafter as the control volumes for the pressure and density, the $x$-component and the $y$-component of the velocity respectively. As usual at the continuous level or for variational discrete formulation (as in the finite element context), the Dirichlet boundary conditions are (partly) incorporated in the definition of the discrete spaces, and, to this purpose, we introduce $S_{0}^{x} \subset S^{x}$ and $S_{0}^{y} \subset S^{y}$ defined as follows:

$$
\begin{aligned}
& S_{0}^{x}=\left\{v^{x} \in S^{x} \text { such that } v^{x}=0 \text { over } K_{\frac{1}{2}, j}^{x} \text { and } K_{N+\frac{1}{2}, j}^{x}, \text { for } 1 \leq j \leq M\right\}, \\
& S_{0}^{y}=\left\{v^{y} \in S^{y} \text { such that } v^{y}=0 \text { over } K_{i, \frac{1}{2}}^{y} \text { and } K_{i, M+\frac{1}{2}}^{y}, \text { for } 1 \leq i \leq N\right\} .
\end{aligned}
$$




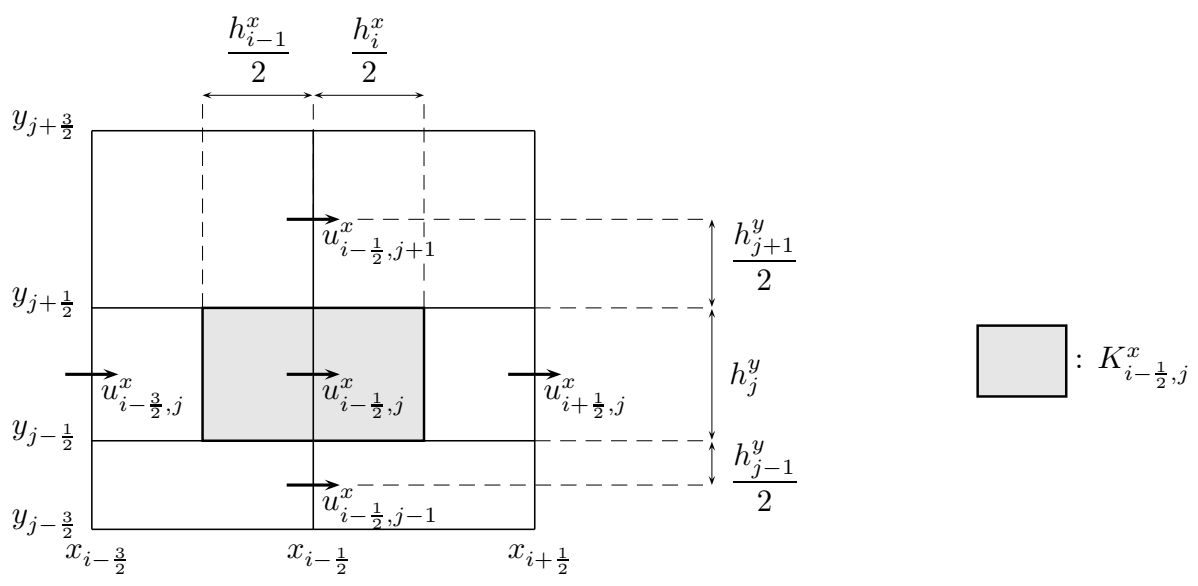

FIG. 3.2. Notations for the control volume $K_{i-\frac{1}{2}, j}^{x}$.

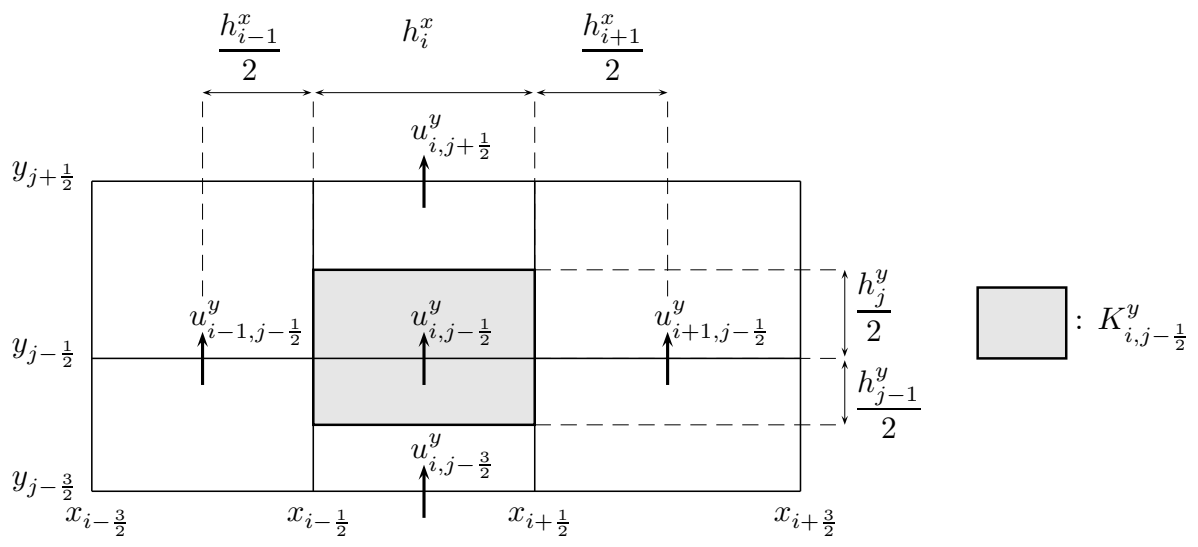

FIG. 3.3. Notations for the control volume $K_{i, j-\frac{1}{2}}^{y}$.

The $x$-component of the velocity will be supposed to belong to $S_{0}^{x}$, which consists in setting to zero the degrees of freedom of $v^{x}$ located at the left boundary (i.e. $\{0\} \times(0,1)$ ) and right boundary (i.e. $\{1\} \times(0,1))$ of $\Omega$; the $y$-component will be supposed to belong to $S_{0}^{y}$, which consists in setting to zero the degrees of freedom of $v^{y}$ located at the bottom boundary $($ i.e. $(0,1) \times\{0\})$ and top boundary $($ i.e. $(0,1) \times\{1\})$ of $\Omega$. Since the discrete functions are determined by the values that they take over their respective control volumes, the unknowns of the discrete problem are:

$$
\begin{aligned}
& p_{i, j}, \rho_{i, j}, 1 \leq i \leq N, 1 \leq j \leq M \\
& u_{i-\frac{1}{2}, j}^{x}, 1 \leq i \leq N+1,1 \leq j \leq M, \quad u_{i, j-\frac{1}{2}}^{y}, 1 \leq i \leq N, 1 \leq j \leq M+1
\end{aligned}
$$

and the Dirichlet boundary conditions imply that $u_{\frac{1}{2}, j}^{x}=u_{N+\frac{1}{2}, j}^{x}=0$ for $1 \leq j \leq M$, and $u_{i, \frac{1}{2}}^{y}=u_{i, M+\frac{1}{2}}^{y}=0$ for $1 \leq i \leq N$.

For each component of the velocity, the finite volume discrete Laplace operator is given by a classical five-point formula. This discrete Laplace operator is obtained 
by writing a discrete momentum flux balance for each component over each corresponding velocity cell $K^{x}$ or $K^{y}$; it is well known that it is not consistent in the finite difference sense, see e.g. [4, Section 5.2 page 732] except on uniform meshes. For the $x$-component of the velocity, the discrete Laplacian is such that $\Delta^{x} v^{x} \in S_{0}^{x}$ for any $v^{x} \in S_{0}^{x}$, and as usual in the finite volume formulation, implicitly takes into account the Dirichlet boundary at the top and bottom boundaries; since $u^{x}$ is prescribed to zero in the left and right layers of control volumes, the Laplace operator is not defined in these control volumes. Similarly, the discrete Laplacian for the $y$-component of the velocity is such that, $\forall v^{y} \in S_{0}^{y}, \Delta^{y} v^{y} \in S_{0}^{y}$, implicitly takes into account the Dirichlet boundary conditions at the left and right side, and does not need to be prescribed in the bottom and top layers of control volumes. Let us now define the following bilinear forms over respectively $S_{0}^{x}$ and $S_{0}^{y}$ :

$$
\begin{aligned}
& \begin{aligned}
\forall v^{x}, w^{x} \in S_{0}^{x}, \\
\left(v^{x}, w^{x}\right)^{x}=\int_{\Omega} \Delta^{x} v^{x} w^{x} \mathrm{~d} \boldsymbol{x}=\sum_{\substack{2 \leq i \leq N, 1 \leq j \leq M}}\left|K_{i-\frac{1}{2}, j}^{x}\right| \Delta^{x} v_{i-\frac{1}{2}, j}^{x} w_{i-\frac{1}{2}, j}^{x}, \\
\forall v^{y}, w^{y} \in S_{0}^{y}, \\
\left(v^{y}, w^{y}\right)^{y}=\int_{\Omega} \Delta^{y} v^{y} w^{y} \mathrm{~d} \boldsymbol{x}=\sum_{\substack{1 \leq i \leq N, 2 \leq j \leq M}}\left|K_{i, j-\frac{1}{2}}^{y}\right| \Delta^{y} v_{i, j-\frac{1}{2}}^{y} w_{i, j-\frac{1}{2}}^{y},
\end{aligned}
\end{aligned}
$$

where $|\cdot|$ stands for the Euclidean measure. Let $\mathcal{E}^{x}$ be the set of the edges of the control volumes $\left(K_{i-\frac{1}{2}, j}^{x}\right)_{1 \leq i \leq N+1,1 \leq j \leq M}$ associated to the $x$-component of the velocity, including the internal ones (i.e. separating two control volumes) and the external ones (i.e. included in the boundary of $\Omega$ ). For each edge $\sigma^{x} \in \mathcal{E}^{x}$ and $v^{x} \in S^{x}$, we denote by $\left[v^{x}\right]_{\sigma^{x}}$ either the jump of $v^{x}$ across $\sigma^{x}$ if $\sigma^{x}$ is an internal edge, or the value of $v^{x}$ in the control volume adjacent to $\sigma^{x}$ if $\sigma^{x}$ is an external edge. Reordering the summation in Equation (3.5), we obtain:

$$
\forall v^{x}, w^{x} \in S_{0}^{x}, \quad\left(v^{x}, w^{x}\right)^{x}=\sum_{\sigma^{x} \in \mathcal{E}^{x}} \frac{\left|\sigma^{x}\right|}{d_{\sigma^{x}}}\left[v^{x}\right]_{\sigma^{x}}\left[w^{x}\right]_{\sigma^{x}},
$$

where, for an internal edge $\sigma^{x}, d_{\sigma^{x}}$ stands for the distance between the mass centers of the two control volumes adjacent to $\sigma^{x}$ and, for an external edge $\sigma^{x}, d_{\sigma^{x}}$ stands for the distance between the mass center of the control volume adjacent to $\sigma^{x}$ and the boundary. With similar notations, we obtain:

$$
\forall v^{y}, w^{y} \in S_{0}^{y}, \quad\left(v^{y}, w^{y}\right)^{y}=\sum_{\sigma^{y} \in \mathcal{E}^{y}} \frac{\left|\sigma^{y}\right|}{d_{\sigma^{y}}}\left[v^{y}\right]_{\sigma^{y}}\left[w^{y}\right]_{\sigma^{y}} .
$$

These bilinear forms are thus symmetric. Moreover each form induces a norm over respectively $S_{0}^{x}$ and $S_{0}^{y}$, which we denote:

$$
\left\|v^{x}\right\|_{x}^{2}=\left(v^{x}, v^{x}\right)^{x}, \quad\left\|v^{y}\right\|_{y}^{2}=\left(v^{y}, v^{y}\right)^{y} .
$$

This norm is known to control the $\mathrm{L}^{2}$ norm by a discrete Poincaré inequality [4, Lemma 9.1 page 765]. In addition, a compactness result holds for sequences of discrete functions bounded with respect to this norm. This result is given in Theorem 3.1 
below; to state it, we first define discrete derivatives of elements of $S_{0}^{x}$ and $S_{0}^{y}$. For $\boldsymbol{v}=\left(v^{x}, v^{y}\right) \in S_{0}^{x} \times S_{0}^{y}$, we define $\partial_{\mathcal{M}}^{x} v^{x} \in S$ and $\partial_{\mathcal{M}}^{y} v^{y} \in S$ by:

$$
\left(\partial_{\mathcal{M}}^{x} v^{x}\right)_{i, j}=\frac{v_{i+\frac{1}{2}, j}^{x}-v_{i-\frac{1}{2}, j}^{x}}{h_{i}^{x}}, \quad\left(\partial_{\mathcal{M}}^{y} v^{y}\right)_{i, j}=\frac{v_{i, j+\frac{1}{2}}^{y}-v_{i, j-\frac{1}{2}}^{y}}{h_{j}^{y}}
$$

in $K_{i, j}, i \in\{1, \ldots, N\}$ and $j \in\{1, \ldots, M\}$. The formulae (3.9) give, in particular, a discrete divergence operator $\operatorname{div}_{\mathcal{M}} \boldsymbol{v}=\partial_{\mathcal{M}}^{x} v^{x}+\partial_{\mathcal{M}}^{y} v^{y}$ (see (3.14)-(3.15) below). In order to define $\partial_{\mathcal{M}}^{y} v^{x}$ and $\partial_{\mathcal{M}}^{x} v^{y}$, we need a fourth partition of $\Omega$ :

$$
\bar{\Omega}=\bigcup_{\substack{1 \leq i \leq N+1 \\ 1 \leq j \leq M+1}} \bar{K}_{i-\frac{1}{2}, j-\frac{1}{2}}^{x y}, \quad \text { with } K_{i-\frac{1}{2}, j-\frac{1}{2}}^{x y}=\left(x_{i-1}, x_{i}\right) \times\left(y_{j-1}, y_{j}\right)
$$

The fourth discrete function space is the space $S^{x y} \subset \mathrm{L}^{\infty}(\Omega)$ which stands for piecewise constant functions over each control volume $K^{x y}$. We now define $\partial_{\mathcal{M}}^{y} v^{x} \in S^{x y}$ and $\partial_{\mathcal{M}}^{x} v^{y} \in S^{x y}$ by, for all $i \in\{0, \ldots, N\}$ and $j \in\{0, \ldots, M\}$ :

$$
\begin{aligned}
& \partial_{\mathcal{M}}^{y} v^{x}=\left(\partial_{\mathcal{M}}^{y} v^{x}\right)_{i+\frac{1}{2}, j+\frac{1}{2}}=\frac{v_{i+\frac{1}{2}, j+1}^{x}-v_{i+\frac{1}{2}, j}^{x}}{h_{j+\frac{1}{2}}^{y}} \text { in } K_{i+\frac{1}{2}, j+\frac{1}{2}}^{x y}, \\
& \partial_{\mathcal{M}}^{x} v^{y}=\left(\partial_{\mathcal{M}}^{x} v^{y}\right)_{i+\frac{1}{2}, j+\frac{1}{2}}=\frac{v_{i+1, j+\frac{1}{2}}^{y}-v_{i, j+\frac{1}{2},}^{y}}{h_{i+\frac{1}{2}}^{x}} \text { in } K_{i+\frac{1}{2}, j+\frac{1}{2}}^{x y},
\end{aligned}
$$

where $h_{j+\frac{1}{2}}^{x}$ and $h_{i+\frac{1}{2}}^{y}$ are defined by (3.3) and

$$
v_{i-\frac{1}{2}, 0}^{x}=0, v_{i-\frac{1}{2}, M+1}^{x}=0, v_{0, j-\frac{1}{2}}^{y}=0, v_{N+1, j-\frac{1}{2}}^{y}=0 .
$$

We may then define a discrete curl operator, useful for the the proof of convergence of the scheme, by $\operatorname{curl}_{\mathcal{M}} \boldsymbol{v}=\partial_{\mathcal{M}}^{x} v^{y}-\partial_{\mathcal{M}}^{y} v^{x}$. Similary, a discrete curl operator may also be defined in the case $d=3$ with an obvious discretization of the continuous curl operator.

TheOREM 3.1. Consider a sequence of MAC grids $\left(\mathcal{M}_{n}\right)_{n \in \mathbb{N}}$, with step size $h^{n}$ (as defined by (3.1)) tending to zero as $n \rightarrow \infty$. Let $\left(u_{n}^{x}\right)_{n \in \mathbb{N}}\left(\right.$ resp. $\left.\left(u_{n}^{y}\right)_{n \in \mathbb{N}}\right)$ be a sequence of discrete functions, i.e. such that each element of the sequence $u_{n}^{x}$ (resp. $u_{n}^{y}$ ) belongs to a space $S^{x, n}$ (resp. $S^{y, n}$ ) and such that $\left\|u_{n}^{x}\right\|_{x}$ (resp. $\left\|u_{n}^{y}\right\|_{y}$ ) is bounded. Then the sequence $\left(u_{n}^{x}\right)_{n \in \mathbb{N}}\left(\right.$ resp. $\left.\left(u_{n}^{y}\right)_{n \in \mathbb{N}}\right)$ converges in $\mathrm{L}^{2}(\Omega)$ to a limit $u^{x}$ (resp. $u^{y}$ ), and this limit satisfies $u^{x} \in \mathrm{H}_{0}^{1}(\Omega)$ (resp. $u^{y} \in \mathrm{H}_{0}^{1}(\Omega)$ ). Furthermore, one has $\left(\partial_{\mathcal{M}_{n}}^{x} u_{n}^{x}, \partial_{\mathcal{M}_{n}}^{y} u_{n}^{x}\right) \rightarrow \nabla u^{x}\left(\right.$ resp. $\left.\left(\partial_{\mathcal{M}_{n}}^{x} u_{n}^{y}, \partial_{\mathcal{M}_{n}}^{y} u_{n}^{y}\right) \rightarrow \nabla u^{y}\right)$ weakly in $L^{2}(\Omega)^{2}$, as $n \rightarrow \infty$.

Proof. The proof of convergence of the sequence $\left(u_{n}^{x}\right)_{n \in \mathbb{N}}$ is similar to that of $[4$, Theorem 9.1 page 772]. The weak convergence of the discrete gradient in $L^{2}(\Omega)^{2}$ is easily obtained by discrete integration by parts, thus shifting the discrete derivatives on a smooth test function and passing to the limit on its discrete derivative.

The discrete $\mathrm{H}^{1}$ norm of a velocity field $\boldsymbol{v}=\left(v^{x}, v^{y}\right) \in S_{0}^{x} \times S_{0}^{y}$ is defined from the $\|\cdot\|_{x}$ norm and the $\|\cdot\|_{y}$ norm of its $x$-component and $y$-component respectively by $\|\boldsymbol{v}\|_{\mathcal{M}}^{2}=\left\|v^{x}\right\|_{x}^{2}+\left\|v^{y}\right\|_{y}^{2}$.

We now define the discrete gradient as follows:

$$
\nabla_{\mathcal{M}}: \mid \begin{aligned}
& S \longrightarrow S_{0}^{x} \times S_{0}^{y} \\
& q \mapsto\left(\left(\nabla_{\mathcal{M}} q\right)^{x},\left(\nabla_{\mathcal{M}} q\right)^{y}\right)
\end{aligned}
$$


with:

$$
\begin{aligned}
& \text { for } 2 \leq i \leq N, 1 \leq j \leq M, \quad\left(\nabla_{\mathcal{M}} q\right)_{i-\frac{1}{2}, j}^{x}=\frac{1}{h_{i-\frac{1}{2}}^{x}}\left(q_{i, j}-q_{i-1, j}\right), \\
& \text { for } 1 \leq i \leq N, 2 \leq j \leq M, \quad\left(\nabla_{\mathcal{M}} q\right)_{i, j-\frac{1}{2}}^{y}=\frac{1}{h_{j-\frac{1}{2}}^{y}}\left(q_{i, j}-q_{i, j-1}\right) .
\end{aligned}
$$

Note that the values of the $x$-component (resp. $y$-component) of the gradient over the left and right (resp. bottom and top) boundary sides of the mesh do not need to be defined, since it is supposed by definition to vanish: $\left(\nabla_{\mathcal{M}} q\right)^{x} \in S_{0}^{x}\left(\operatorname{resp} .\left(\nabla_{\mathcal{M}} q\right)^{y} \in S_{0}^{y}\right)$. Let us now define a discrete divergence operator as follows:

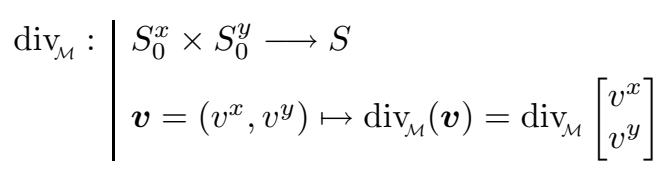

with, for $1 \leq i \leq N, 1 \leq j \leq M$ :

$$
\operatorname{div}_{\mathcal{M}}\left[\begin{array}{l}
v^{x} \\
v^{y}
\end{array}\right]_{i, j}=\frac{1}{h_{i}^{x} h_{j}^{y}}\left(h_{j}^{y} v_{i+\frac{1}{2}, j}^{x}+h_{i}^{x} v_{i, j+\frac{1}{2}}^{y}-h_{j}^{y} v_{i-\frac{1}{2}, j}^{x}-h_{i}^{x} v_{i, j-\frac{1}{2}}^{y}\right) .
$$

This divergence operator satisfies:

$$
\forall \boldsymbol{v} \in S_{0}^{x} \times S_{0}^{y}, \quad\left\|\operatorname{div}_{\mathcal{M}}(\boldsymbol{v})\right\|_{\mathrm{L}^{2}(\Omega)} \leq \sqrt{2}\|\boldsymbol{v}\|_{\mathcal{M}} .
$$

A fundamental feature of the MAC scheme is that the discrete gradient operator and the (opposite of the) divergence operator are dual with respect to the $\mathrm{L}^{2}$ inner product:

$$
\forall \boldsymbol{v} \in S_{0}^{x} \times S_{0}^{y}, \forall q \in S, \quad \int_{\Omega} \boldsymbol{v} \cdot \nabla_{\mathcal{M}} q \mathrm{~d} \boldsymbol{x}=-\int_{\Omega} q \operatorname{div}_{\mathcal{M}}(\boldsymbol{v}) \mathrm{d} \boldsymbol{x} .
$$

Another fundamental feature of the scheme is its so-called "inf-sup" stability property $[18,21,19]$, which reads:

$$
\forall q \in S, \quad \sup _{\boldsymbol{v} \in S_{0}^{x} \times S_{0}^{y}} \frac{\int_{\Omega} q \operatorname{div}_{\mathcal{M}}(\boldsymbol{v}) \mathrm{d} \boldsymbol{x}}{\|\boldsymbol{v}\|_{\mathcal{M}}} \geq c_{\mathrm{is}}\|q-m(q)\|_{\mathrm{L}^{2}(\Omega)} .
$$

where $c_{\text {is }}$ is a real number only depending on $\Omega$ and the parameter $\eta$ characterizing the uniformity of the mesh, and $m(q)$ denotes the mean value of $q$ over $\Omega$.

4. Presentation of the scheme and of the main results. Consider a MAC grid as defined in the previous section, which we denote by $\mathcal{M}$. A crucial property which must be satisfied by the scheme we are about to construct is that the resulting approximate density stays positive. To this purpose, the mass equation (2.1b) is discretized with an upwind choice for the density. For any $\rho \in S$ and $\boldsymbol{v}=\left(v^{x}, v^{y}\right) \in$ $S^{x} \times S^{y}$, we define an upwind discrete divergence $\operatorname{div}_{\mathcal{M}}^{\text {up }}(\rho \boldsymbol{v}) \in S$ defined by its values on each cell $K_{i, j}$ :

$$
\begin{aligned}
\left(\operatorname{div}_{\mathcal{M}}^{\mathrm{up}}(\rho \boldsymbol{v})\right)_{i, j}=\frac{1}{h_{i}^{x} h_{j}^{y}}\left(h_{j}^{y} \rho_{i+\frac{1}{2}, j} v_{i+\frac{1}{2}, j}^{x}\right. & +h_{i}^{x} \rho_{i, j+\frac{1}{2}} v_{i, j+\frac{1}{2}}^{y} \\
& \left.-h_{j}^{y} \rho_{i-\frac{1}{2}, j} v_{i-\frac{1}{2}, j}^{x}-h_{i}^{x} \rho_{i, j-\frac{1}{2}} v_{i, j-\frac{1}{2}}^{y}\right)
\end{aligned}
$$


where the value of the density at each edge is upwinded with respect to the velocity:

$$
\mid \begin{aligned}
& \rho_{i+\frac{1}{2}, j}=\rho_{i, j} \text { if } v_{i+\frac{1}{2}, j}^{x} \geq 0 \text { and } \rho_{i+\frac{1}{2}, j}=\rho_{i+1, j} \text { otherwise, } \\
& \rho_{i, j+\frac{1}{2}}=\rho_{i, j} \text { if } v_{i, j+\frac{1}{2}}^{y} \geq 0 \text { and } \rho_{i, j+\frac{1}{2}}=\rho_{i, j+1} \text { otherwise. }
\end{aligned}
$$

We define an average density $\rho^{*}$ by $\rho^{*}=M /|\Omega|$, and $\left(f^{x}, f^{y}\right) \in S^{x} \times S^{y}$ by:

$$
\begin{array}{ll}
\left(f^{x}\right)_{i-\frac{1}{2}, j}=\frac{1}{\left|K_{i-\frac{1}{2}, j}^{x}\right|} \int_{K_{i-\frac{1}{2}, j}^{x}} f^{x} \mathrm{~d} \boldsymbol{x} & \text { for } 2 \leq i \leq N, 1 \leq j \leq M, \\
\left(f^{y}\right)_{i, j-\frac{1}{2}}=\frac{1}{\left|K_{i, j-\frac{1}{2}}^{y}\right|} \int_{K_{i, j-\frac{1}{2}}^{y}}^{y} f^{y} \mathrm{~d} \boldsymbol{x} & \text { for } 1 \leq i \leq N, 2 \leq j \leq M .
\end{array}
$$

REMARK 4.1. The density $\rho^{*}$ can be chosen as any positive function satisfying $\rho^{*} \in S$ and $\int_{\Omega} \rho^{*} \mathrm{~d} \boldsymbol{x}=M$. However, the solutions of the scheme may depend on the choice of $\rho^{*}$.

With these notations, the MAC scheme reads, for a given positive $\alpha$ :

Find $\boldsymbol{u}=\left(u^{x}, u^{y}\right)^{t} \in S_{0}^{x} \times S_{0}^{y}, p \in S$ and $\rho \in S$ such that:

$$
\begin{aligned}
& \left(\Delta^{x} u^{x}\right)_{i-\frac{1}{2}, j}+\left(\left(\nabla_{\mathcal{M}} p\right)^{x}\right)_{i-\frac{1}{2}, j}=\left(f^{x}\right)_{i-\frac{1}{2}, j}, 2 \leq i \leq N, 1 \leq j \leq M, \\
& \left(\Delta^{y} u^{y}\right)_{i, j-\frac{1}{2}}+\left(\left(\nabla_{\mathcal{M}} p\right)^{y}\right)_{i, j-\frac{1}{2}}=\left(f^{y}\right)_{i, j-\frac{1}{2}}, 1 \leq i \leq N, 2 \leq j \leq M, \\
& \operatorname{div}_{\mathcal{M}}^{\text {up }}(\rho \boldsymbol{u})+h^{\alpha}\left(\rho-\rho^{*}\right)=0 \\
& p=\rho^{\gamma}, \rho>0 .
\end{aligned}
$$

Note that this scheme conserves the total mass; indeed, summing (4.2c) over $K \in \mathcal{M}$ yields that the integral of $\rho$ and $\rho^{*}$ over $\Omega$ are equal.

From the identities (3.5) and (3.16), the first two equations of the scheme may be set under the following discrete variational form:

$$
\begin{aligned}
& \boldsymbol{u}=\left(u^{x}, u^{y}\right) \in S_{0}^{x} \times S_{0}^{y}, p \in S, \text { and, } \forall \boldsymbol{v}=\left(v^{x}, v^{y}\right) \in S_{0}^{x} \times S_{0}^{y} \\
& \left(u^{x}, v^{x}\right)_{x}+\left(u^{y}, v^{y}\right)_{y}-\int_{\Omega} p \operatorname{div}_{\mathcal{M}} \boldsymbol{v} \mathrm{d} \boldsymbol{x}=\int_{\Omega} \boldsymbol{f} \cdot \boldsymbol{v} \mathrm{d} \boldsymbol{x} .
\end{aligned}
$$

In Section 5 below we prove the existence of a solution to the scheme 4.2, along with some estimates. The remainder of the paper is devoted to the proof of the following convergence theorem.

THEOREM 4.1 (Convergence of the MAC scheme). Let $\Omega$ be an open bounded subset of $\mathbb{R}^{d}, d=2$ or $d=3$, adapted to the $M A C$-scheme (that is any finite union of rectangles in $2 D$ or rectangular parallelipeds in $3 D)$. Let $\boldsymbol{f} \in\left(\mathrm{L}^{2}(\Omega)\right)^{d}, M>0, \gamma>1$, and $\alpha>0$. Let $\eta>0$ and $\left(\mathcal{M}_{n}\right)_{n \in \mathbb{N}}$ be a sequence of MAC grids satisfying (3.2) and with step size $h^{n}$ tending to zero as $n \rightarrow \infty$. Let $\left(\boldsymbol{u}_{n}\right)_{n \in \mathbb{N}},\left(\rho_{n}\right)_{n \in \mathbb{N}}$ and $\left(p_{n}\right)_{n \in \mathbb{N}}$ be the corresponding sequence of MAC-discrete solutions. Then, up to the extraction of a subsequence:

- the sequence $\left(\boldsymbol{u}_{n}\right)_{n \in \mathbb{N}}$ converges in $\left(\mathrm{L}^{2}(\Omega)\right)^{d}$ to a function $\boldsymbol{u} \in \mathrm{H}_{0}^{1}(\Omega)^{d}$,

- the sequence $\left(\rho_{n}\right)_{n \in \mathbb{N}}$ converges in $\mathrm{L}^{p}(\Omega)$ for any $p$ such that $1 \leq p<2 \gamma$ and weakly in $\mathrm{L}^{2 \gamma}(\Omega)$ to a function $\rho$ of $\mathrm{L}^{2 \gamma}(\Omega)$,

- the sequence $\left(p_{n}\right)_{n \in \mathbb{N}}$ converges in $\mathrm{L}^{p}(\Omega)$ for any $p$ such that $1 \leq p<2$ and weakly in $\mathrm{L}^{2}(\Omega)$ to a function $p$ of $\mathrm{L}^{2}(\Omega)$, 
- $\boldsymbol{u}, \rho$ and $p$ satisfy the continuous problem (2.2).

REMARK 4.2. The same convergence result holds (with no problem of passage to the limit in the equation of state) for $\gamma=1$, with only a weak convergence of $\left(\rho_{n}\right)_{n \in \mathbb{N}}$ and $\left(p_{n}\right)_{n \in \mathbb{N}}$ in $\mathrm{L}^{2}(\Omega)$.

5. Existence of the solution and estimates. To avoid the use of the indexes $i, j$ when possible, we use in this section notations designed for general grids (see e.g. [7]). We denote by $\mathcal{E}_{\text {int }}$ the set of all the internal edges $\sigma=K \mid L$ of the (pressure) grid, where $K$ and $L$ are the two (pressure) control volumes separated by $\sigma$. We then define a normal vector to any edge of the pressure grid by $\boldsymbol{n}_{\sigma}=(1,0)^{t}$ or $\boldsymbol{n}_{\sigma}=(0,1)^{t}$. For any $K \in \mathcal{M}$, we denote by $\mathcal{E}_{K}$ the set of edges of $K$ and for any $\sigma \in \mathcal{E}_{K}$, we introduce $\epsilon_{\sigma}^{K}=\boldsymbol{n}_{\sigma}^{K} \cdot \boldsymbol{n}_{\sigma}$ where $\boldsymbol{n}_{\sigma}^{K}$ denotes the unit normal vector to $\sigma$ outward to $K$ (so that $\epsilon_{\sigma}^{K}= \pm 1$ ). For a discrete velocity field $\boldsymbol{u} \in S_{0}^{x} \times S_{0}^{y}$, we denote by $u_{\sigma}$ the unknown associated to the edge $\sigma$, that is $u_{\sigma}=u_{i+\frac{1}{2}, j}^{x}$. (resp. $u_{\sigma}=u_{i, j+\frac{1}{2}}^{x}$ ) if $\sigma$ is the edge separating $K_{i, j}$ from $K_{i+1, j}$ (resp. $K_{i, j+1}$ ). Then, for a density field $\rho \in S$ and an internal edge $\sigma=K \mid L$, we denote by $\rho_{\sigma}$ the upwind choice for $\rho$ on $\sigma$ with respect to $\boldsymbol{u}$, that is $\rho_{\sigma}=\rho_{K}$ if $\boldsymbol{u} \cdot \boldsymbol{n}_{\sigma}^{K} \geq 0, \rho_{\sigma}=\rho_{L}$ otherwise; finally, $[\rho]_{\sigma}=\left|\rho_{K}-\rho_{L}\right|$. With these notations, the discrete divergence given in (3.15) becomes:

$$
\operatorname{div}_{\mathcal{M}} \boldsymbol{u}=\sum_{\sigma \in \mathcal{E}_{K}}|\sigma| u_{\sigma} \epsilon_{\sigma}^{K}
$$

and similarly, the discrete upwind divergence given in (4.1) becomes:

$$
\operatorname{div}_{\mathcal{M}}^{\mathrm{up}}(\rho \boldsymbol{u})=\sum_{\sigma \in \mathcal{E}_{K}}|\sigma| \rho_{\sigma} u_{\sigma} \epsilon_{\sigma}^{K} .
$$

The following lemma states the positivity of the discrete density. It is (easily) proven by observing that for a given velocity field, the discrete mass balance (4.2c) is a linear system for $\rho$ the matrix of which is an M-matrix [8, Lemma 2.1].

LEMmA 5.1. Let $u^{x} \in S_{0}^{x}, u^{y} \in S_{0}^{y}$ and $\rho \in S$ satisfy (4.2c). Then $\rho>0$.

Proposition 5.2. There exists a solution to System (4.2).

Proof. The proof of this result is obtained by applying the fixed point Brouwer theorem. Let $\boldsymbol{u}^{k}=\left(\left(u^{x}\right)^{k},\left(u^{y}\right)^{k}\right)^{t} \in S_{0}^{x} \times S_{0}^{y}$ be a given discrete velocity field. We define $\rho^{k+1}$ as the solution of (4.2c) with $u^{x}=\left(u^{x}\right)^{k}$ and $u^{y}=\left(u^{y}\right)^{k}$, i.e.:

$$
\operatorname{div}_{\mathcal{M}}^{\text {up }}\left(\rho^{k+1} \boldsymbol{u}^{k}\right)+h^{\alpha}\left(\rho^{k+1}-\rho^{*}\right)=0 .
$$

The existence of a solution to this linear system is a consequence of Lemma 5.1 along with the positivity of this solution. In addition, we deduce from the conservativity of the scheme that:

$$
\int_{\Omega} \rho^{k+1} \mathrm{~d} \boldsymbol{x}=\int_{\Omega} \rho^{*} \mathrm{~d} \boldsymbol{x} .
$$

This relation provides a bound for $\rho^{k+1}$ in the $\mathrm{L}^{1}$ norm, and therefore in all norms since the problem is of finite dimension. Through the equation of state, we thus obtain a bound for $p^{k+1}=\left(\rho^{k+1}\right)^{\gamma}$ in a discrete norm. We now define $\left(u^{x}\right)^{k+1}$ and $\left(u^{y}\right)^{k+1}$ as the solution, with $p=p^{k+1}$, of $(4.2 \mathrm{a})$ and $(4.2 \mathrm{~b})$ respectively:

$$
\begin{aligned}
& \left(\Delta^{x}\left(u^{x}\right)^{k+1}\right)_{i-\frac{1}{2}, j}+\left(\left(\nabla_{\mathcal{M}} p^{k+1}\right)^{x}\right)_{i-\frac{1}{2}, j}=\left(f^{x}\right)_{i-\frac{1}{2}, j}, 2 \leq i \leq N, 1 \leq j \leq M, \\
& \left(\Delta^{y}\left(u^{y}\right)^{k+1}\right)_{i, j-\frac{1}{2}}+\left(\left(\nabla_{\mathcal{M}} p^{k+1}\right)^{y}\right)_{i, j-\frac{1}{2}}=\left(f^{y}\right)_{i, j-\frac{1}{2}}, 1 \leq i \leq N, 2 \leq j \leq M .
\end{aligned}
$$


Since $p^{k+1}$ is bounded, its gradient $\nabla_{\mathcal{M}} p^{k+1}$ is bounded, in particular in $\mathrm{L}^{2}(\Omega)^{2}$ (note, however, that the bounds that we thus obtain depend on the mesh). Multiplying (4.2a) by $\left(\left(u^{x}\right)^{k+1}\right)_{i-\frac{1}{2}, j}$, summing over $i$ and $j$, and using (3.5), we get:

$$
\left\|\left(u^{x}\right)^{k+1}\right\|_{x}^{2}=\int_{\Omega}\left[-\left(\nabla_{\mathcal{M}} p^{k+1}\right)^{x}+f^{x}\right]\left(u^{x}\right)^{k+1} \mathrm{~d} \boldsymbol{x} .
$$

Invoking now the fact that the $\|\cdot\|_{x}$ norm controls the $\mathrm{L}^{2}$ norm, we thus obtain an a priori bound for $\left\|\left(u^{x}\right)^{k+1}\right\|_{x}$, let us say $\left\|\left(u^{x}\right)^{k+1}\right\|_{x} \leq C^{x}$, with $C^{x}$ depending only on $f^{x}$ and $\left(\nabla_{\mathcal{M}} p^{k+1}\right)^{x}$. Taking $f^{x}=0$ and $\left(p^{k+1}\right)^{x}=0$, this estimate provides the uniqueness of $\left(u^{x}\right)^{k+1}$ solution to (4.2a) and in turn, its existence. The same arguments hold for $\left(u^{y}\right)^{k+1}$, and we thus get that $\left(u^{y}\right)^{k+1}$ exists and satisfies an inequality of the form $\left\|\left(u^{y}\right)^{k+1}\right\|_{y} \leq C^{y}$. Finally, the function $\left.\left(\left(u^{x}\right)^{k},\left(u^{y}\right)^{k}\right)\right) \mapsto$ $\left.\left(\left(u^{x}\right)^{k+1},\left(u^{y}\right)^{k+1}\right)\right)$ is continuous. By the Brouwer theorem, it thus admits a fixed point in the convex set $\mathcal{C}$ defined by:

$$
\mathcal{C}=\left\{\left(v^{x}, v^{y}\right) \in S_{0}^{x} \times S_{0}^{y} \text { s.t. }\left\|u^{x}\right\|_{x} \leq C^{x} \text { and }\left\|u^{y}\right\|_{y} \leq C^{y}\right\} .
$$

This fixed point is a solution to the scheme.

Let us now turn to stability issues: in order to prove convergence, we wish to obtain some uniform (with respect to the mesh) bounds on the solutions to (4.2); these bounds are stated in Lemma 5.5 below. We begin by a technical lemma, which extends [5, Lemma 5.1] and yields Lemma 5.4. In fact, Lemma 5.4 is not only useful for stability issues, but for three other reasons. Firstly, it allows an estimate on $\boldsymbol{u}$ in a dicrete $\mathrm{H}_{0}^{1}$ norm (Proposition 5.5), as in [5]. Secondly, it yields a so called weak BV estimate if $\gamma>2$ or weighted weak BV estimate if $\gamma \leq 2$ (Proposition 5.5). These weak BV estimates depend on the mesh and do not give a direct compactness result on the sequence of approximate solutions; however they are useful in the passage to the limit in the mass equation. Note that the weak (and weighted weak) BV estimate did not have to be used in [5] because of the presence of an additional stabilization term; this latter term was in fact introduced for technical reasons for the passage to the limit in the equation of state. Thirdly, Lemma 5.4 gives (with $\beta=1$ ) a crucial inequality which is also used in order to pass to the limit in the equation of state.

Lemma 5.3. Let $\rho$ be a positive function of $S$, and $\boldsymbol{u}=\left(u^{x}, u^{y}\right) \in S_{0}^{x} \times S_{0}^{y}$. Let $\varphi$ be a continuously twice-differentiable function from $(0,+\infty)$ to $\mathbb{R}$. Then, for any $\sigma \in \mathcal{E}_{\text {int }}, \sigma=K \mid L$, there exists a real number $\tilde{\rho}_{\sigma} \in\left[\min \left(\rho_{K}, \rho_{L}\right), \max \left(\rho_{K}, \rho_{L}\right)\right]$ such that:

$$
\int_{\Omega} \varphi^{\prime}(\rho) \operatorname{div}_{\mathcal{M}}^{\mathrm{up}}(\rho \boldsymbol{u}) \mathrm{d} \boldsymbol{x}=\int_{\Omega}\left[\rho \varphi^{\prime}(\rho)-\varphi(\rho)\right] \operatorname{div}_{\mathcal{M}} \boldsymbol{u} \mathrm{d} \boldsymbol{x}+\frac{1}{2} \sum_{\sigma \in \mathcal{E}_{\text {int }}}|\sigma| \varphi^{\prime \prime}\left(\tilde{\rho}_{\sigma}\right)\left|u_{\sigma}\right|[\rho]_{\sigma}^{2} .
$$

Proof. Using the expression of the divergence operator, we get:

$$
\int_{\Omega} \varphi^{\prime}(\rho) \operatorname{div}_{\mathcal{M}}^{\mathrm{up}}(\rho \boldsymbol{u}) \mathrm{d} \boldsymbol{x}=\int_{\Omega}\left[\rho \varphi^{\prime}(\rho)-\varphi(\rho)\right] \operatorname{div}_{\mathcal{M}} \boldsymbol{u} \mathrm{d} \boldsymbol{x}+R,
$$

with:

$$
R=\sum_{K \in \mathcal{M}} \sum_{\sigma \in \mathcal{E}_{K}}|\sigma|\left[\varphi^{\prime}\left(\rho_{K}\right) \rho_{\sigma}-\varphi^{\prime}\left(\rho_{K}\right) \rho_{K}+\varphi\left(\rho_{K}\right)\right] u_{\sigma} \epsilon_{\sigma}^{K}
$$


Reordering the summations and noting that $\epsilon_{\sigma}^{K}=-\epsilon_{\sigma}^{L}$ for $\sigma=K \mid L$, we get:

$$
R=\sum_{\substack{\sigma \in \mathcal{E}_{\text {int }} \\ \sigma=K \mid L}}|\sigma|\left[\left[\varphi^{\prime}\left(\rho_{K}\right) \rho_{\sigma}-\varphi^{\prime}\left(\rho_{K}\right) \rho_{K}+\varphi\left(\rho_{K}\right)\right]-\left[\varphi^{\prime}\left(\rho_{L}\right) \rho_{\sigma}-\varphi^{\prime}\left(\rho_{L}\right) \rho_{L}+\varphi\left(\rho_{L}\right)\right]\right] u_{\sigma} \epsilon_{\sigma}^{K} .
$$

Without loss of generality, we may orient any edge $\sigma \in \mathcal{E}_{\text {int }}$ as $\sigma=K \mid L$ where $K$ is such that $\boldsymbol{u} \cdot \boldsymbol{n}_{\sigma}^{K} \geq 0$, so that $\rho_{\sigma}=\rho_{K}$, and we get:

$$
R=\sum_{\sigma=K \mid L}\left[\varphi\left(\rho_{K}\right)-\varphi\left(\rho_{L}\right)-\varphi^{\prime}\left(\rho_{L}\right)\left(\rho_{K}-\rho_{L}\right)\right]\left|u_{\sigma}\right|,
$$

which yields the result thanks to a Taylor expansion of $\varphi$.

Lemma 5.4. Let $\rho \in S$ and $\boldsymbol{u}=\left(u^{x}, u^{y}\right) \in S_{0}^{x} \times S_{0}^{y}$ satisfy (4.2c). Then, for any $\sigma \in \mathcal{E}_{\text {int }}, \sigma=K \mid L$, for any $\beta \geq 1$ :

$$
\int_{\Omega} \rho^{\beta} \operatorname{div}_{\mathcal{M}} \boldsymbol{u} \mathrm{d} \boldsymbol{x}+\frac{1}{2} \sum_{\sigma \in \mathcal{E}_{\text {int }}} \beta|\sigma| \rho_{\sigma, \beta}\left|u_{\sigma}\right|[\rho]_{\sigma}^{2} \leq C h^{\alpha},
$$

where $C$ only depends on $M, \beta$ and $\Omega$, and

$$
\rho_{\sigma, \beta}=\min \left(\rho_{K}^{\beta-2}, \rho_{L}^{\beta-2}\right) \text { for } \sigma=K \mid L .
$$

Proof. By Lemma 5.1, we know that $\rho>0$. Let us first suppose that $\beta>1$. Multiplying (4.2c) by the test function $\frac{\beta}{\beta-1} \rho^{\beta-1}$ and integrating over $\Omega$, we get:

$$
\frac{\beta}{\beta-1} \int_{\Omega} \rho^{\beta-1} \operatorname{div}_{\mathcal{M}}^{\mathrm{up}}(\rho \boldsymbol{u}) \mathrm{d} \boldsymbol{x}+\frac{\beta h^{\alpha}}{\beta-1} \int_{\Omega} \rho^{\beta-1}\left(\rho-\rho^{*}\right) \mathrm{d} \boldsymbol{x}=0 .
$$

By Lemma 5.3 applied with $\varphi(s)=s^{\beta}$, there exists a family of real numbers $\left(\tilde{\rho}_{\sigma}\right)_{\sigma \in \mathcal{E}_{\text {int }}}$ such that $\tilde{\rho}_{\sigma} \in\left[\min \left(\rho_{K}, \rho_{L}\right), \max \left(\rho_{K}, \rho_{L}\right)\right]$ and such that the first term of Relation (5.5) satisfies:

$$
\frac{\beta}{\beta-1} \int_{\Omega} \rho^{\beta-1} \operatorname{div}_{\mathcal{M}}^{\text {up }}(\rho \boldsymbol{u}) \mathrm{d} \boldsymbol{x}=\int_{\Omega} \rho^{\beta} \operatorname{div}_{\mathcal{M}} \boldsymbol{u} \mathrm{d} \boldsymbol{x}+\frac{1}{2} \sum_{\sigma \in \mathcal{E}_{\text {int }}} \beta|\sigma| \tilde{\rho}_{\sigma}^{\beta-2}\left|u_{\sigma}\right|[\rho]_{\sigma}^{2} .
$$

Since the function $s \mapsto s^{\beta}$ is convex, the second term of Relation (5.5) satisfies:

$$
\frac{\beta}{\beta-1} h^{\alpha} \int_{\Omega} \rho^{\beta-1}\left(\rho-\rho^{*}\right) \mathrm{d} \boldsymbol{x} \geq \frac{h^{\alpha}}{\beta-1} \int_{\Omega}\left(\rho^{\beta}-\left(\rho^{*}\right)^{\beta}\right) \mathrm{d} \boldsymbol{x} \geq \frac{h^{\alpha}}{\beta-1} \int_{\Omega}-\left(\rho^{*}\right)^{\beta} \mathrm{d} \boldsymbol{x} .
$$

The two above relationships and the fact that $\rho_{\sigma, \beta} \leq \tilde{\rho}_{\sigma}^{\beta-2}$ yield the result for $\beta>1$. For $\beta=1$, the arguments are the same with $\varphi(s)=s \log (s)$.

Proposition 5.5. Let $\gamma \geq 1, p \in S, \rho \in S$ and $\boldsymbol{u}=\left(u^{x}, u^{y}\right) \in S_{0}^{x} \times S_{0}^{y}$ be a solution to the scheme, i.e. system (4.2). Then there exists $C_{1}$ depending only on $\boldsymbol{f}$, $M, \Omega$ and $\gamma$ such that:

$$
\|\boldsymbol{u}\|_{\mathcal{M}}+\|p\|_{\mathrm{L}^{2}(\Omega)}+\|\rho\|_{\mathrm{L}^{2 \gamma}(\Omega)} \leq C_{1} .
$$


Moreover, for any $\beta \in[1, \gamma]$, there exists $C_{2}$ depending only on $\boldsymbol{f}, M, \Omega, \gamma$ and $\beta$ such that

$$
\sum_{\sigma \in \mathcal{E}_{\text {int }}}|\sigma| \rho_{\sigma, \beta}\left|u_{\sigma}\right|[\rho]_{\sigma}^{2} \leq C_{2},
$$

where $\rho_{\sigma, \beta}$ is defined in (5.4). In particular, for $\gamma \geq 2$, we get by taking $\beta=2$ in (5.7):

$$
\sum_{\sigma \in \mathcal{E}_{\mathrm{int}}}|\sigma|\left|u_{\sigma}\right|[\rho]_{\sigma}^{2} \leq C_{2}
$$

Finally, $\rho>0, p=\rho^{\gamma}$ and $\int_{\Omega} \rho \mathrm{d} \boldsymbol{x}=M$.

Proof. Let us first notice that $\rho>0$ thanks to Lemma 5.1, that $p=\rho^{\gamma}$ by definition of the scheme (4.2) and that $\int_{\Omega} \rho \mathrm{d} \boldsymbol{x}=M$ thanks to the conservativity of the scheme, see (5.3). By (4.3), we have:

$$
\|\boldsymbol{u}\|_{\mathcal{M}}^{2}-\int_{\Omega} p \operatorname{div}_{\mathcal{M}}(\boldsymbol{u}) \mathrm{d} \boldsymbol{x}=\int_{\Omega}\left(f^{x} u^{x}+f^{y} u^{y}\right) \mathrm{d} \boldsymbol{x},
$$

and thus, thanks to the fact that the discrete $\mathrm{H}^{1}$ norm controls the $\mathrm{L}^{2}$ norm:

$$
\|\boldsymbol{u}\|_{\mathcal{M}}^{2}-\int_{\Omega} p \operatorname{div}_{\mathcal{M}}(\boldsymbol{u}) \mathrm{d} \boldsymbol{x} \leq C_{3},
$$

where $C_{3}$ only depends on $\boldsymbol{f}$ and $\Omega$. Lemma 5.4 with $\beta=\gamma$ yields, since $p=\rho^{\gamma}$ :

$$
\int_{\Omega} p \operatorname{div}_{\mathcal{M}}(\boldsymbol{u}) \mathrm{d} \boldsymbol{x}+\frac{1}{2} \sum_{\sigma \in \mathcal{E}_{\text {int }}} \gamma|\sigma| \rho_{\sigma, \gamma}\left|u_{\sigma}\right|[\rho]_{\sigma}^{2} \leq C_{4},
$$

where $C_{4}$ only depends on $M, \gamma, \alpha$ and $\Omega$. Summing these two relations, we thus obtain:

$$
\|\boldsymbol{u}\|_{\mathcal{M}}^{2}+\frac{1}{2} \sum_{\sigma \in \mathcal{E}_{\text {int }}} \gamma|\sigma| \rho_{\sigma, \gamma}\left|u_{\sigma}\right|[\rho]_{\sigma}^{2} \leq C_{3}+C_{4}
$$

Let $m(p)$ stand for the mean value of $p$. Thanks to the discrete inf-sup condition (3.17), there exists a function $\boldsymbol{v} \in S_{0}^{x} \times S_{0}^{y}, \boldsymbol{v} \neq 0$, such that $\int_{\Omega} p \operatorname{div}_{\mathcal{M}}(\boldsymbol{v}) \mathrm{d} \boldsymbol{x} \geq$ $c_{\text {is }}\|p-m(p)\|_{\mathrm{L}^{2}(\Omega)}\|\boldsymbol{v}\|_{\mathcal{M}}$. Choosing this specific function $\boldsymbol{v}$ as test function in (4.2) then allows a control of $\|p-m(p)\|_{\mathrm{L}^{2}(\Omega)}$. In addition, the positivity of the density and the conservativity of the scheme yields:

$$
\int_{\Omega} \rho \mathrm{d} \boldsymbol{x}=\int_{\Omega} p^{1 / \gamma} \mathrm{d} \boldsymbol{x}=M
$$

and thus a classical result (see e.g. [5, Lemma B.6]) implies that $\|p\|_{\mathrm{L}^{2}(\Omega)}$ itself is bounded, and therefore so is $\|\rho\|_{\mathrm{L}^{2 \gamma}(\Omega)}$. We have therefore proved (5.6) and (5.7) for $\beta=\gamma$.

In order to prove (5.7) for $\beta<\gamma$, let us use once again Lemma 5.4, to obtain:

$$
\sum_{\sigma \in \mathcal{E}_{\text {int }}}|\sigma| \rho_{\sigma, \beta}\left|u_{\sigma}\right|[\rho]_{\sigma}^{2} \leq-\int_{\Omega} \rho^{\beta} \operatorname{div}_{\mathcal{M}}(\boldsymbol{u}) \mathrm{d} \boldsymbol{x}+C,
$$


where $C$ depends on $M, \Omega, \gamma$ and $\beta$.

Since $\rho$ is bounded in $\mathrm{L}^{2 \beta}(\Omega)$ and $\left\|\operatorname{div}_{\mathcal{M}}(\boldsymbol{u})\right\|_{\mathrm{L}^{2}(\Omega)}$ is controlled by $\|\boldsymbol{u}\|_{\mathcal{M}}$, this concludes the proof.

REMARK 5.1 (Strong and weak BV estimate). Note that the estimate (5.8) is the classical weak BV estimate used in the multi-dimensional hyperbolic theory (see e.g. [4, Lemma 21.3 page 889]). It is different from strong BV estimates used in the one-dimensional setting (and which are not available in the multidimensional case for non Cartesian meshes) because it is not independent of the mesh: it behaves as $h^{-\frac{1}{2}}$ where $h$ is the mesh size, but this weak control is still sufficient to pass to the limit in the schemes.

6. Passage to the limit in the mass and momentum balance equations. Proposition 6.1. Let $\eta>0$ and $\left(\mathcal{M}_{n}\right)_{n \in \mathbb{N}}$ be a sequence of MAC grids $\left(\mathcal{M}_{n}\right)_{n \in \mathbb{N}}$ satisfying (3.2) and with step size $h^{n}$ tending to zero as $n \rightarrow \infty$. Let $\left(\boldsymbol{u}_{n}\right)_{n \in \mathbb{N}},\left(\rho_{n}\right)_{n \in \mathbb{N}}$ and $\left(p_{n}\right)_{n \in \mathbb{N}}$ be the corresponding sequence of solutions to (4.3),(4.2c),(4.2d). Then, up to the extraction of a subsequence:

1. the sequence $\left(\boldsymbol{u}_{n}\right)_{n \in \mathbb{N}}$ converges in $\left(\mathrm{L}^{2}(\Omega)\right)^{2}$ to a function $\boldsymbol{u} \in\left(\mathrm{H}_{0}^{1}(\Omega)\right)^{2}$.

2. the sequence $\left(\rho_{n}\right)_{n \in \mathbb{N}}$ weakly converges to a function $\rho$ in $\mathrm{L}^{2 \gamma}(\Omega)$,

3. the sequence $\left(p_{n}\right)_{n \in \mathbb{N}}$ weakly converges to a function $p$ in $\mathrm{L}^{2}(\Omega)$,

4. $\boldsymbol{u}, \rho$ and $p$ satisfy the continuous momentum and mass balance equations $(2.2 \mathrm{a})$ and $(2.2 \mathrm{~b})$.

5. $\rho \geq 0$ a.e. and $\int_{\Omega} \rho \mathrm{d} \boldsymbol{x}=M$.

Proof. The stated convergences (i.e. points 1. to 3.) are straightforward consequences of the uniform bounds for the sequence of solutions, together, for the velocity, with the compactness theorem 3.1. Point 5. is an easy consequence of point 2. Let us then prove point 4. i.e. that $\boldsymbol{u}, \rho$ and $p$ satisfy (2.2a) and (2.2b). Let $\boldsymbol{\varphi}$ be a function of $\mathrm{C}_{c}^{\infty}(\Omega)^{2}$, and let $\varphi_{n}^{x}$ and $\varphi_{n}^{y}$ be the functions of $S^{x, n}$ and $S^{y, n}$ respectively, obtained by taking the average value of $\varphi^{x}$ and $\varphi^{y}$ over each internal edge of the primal mesh $\mathcal{M}_{n}$. Let $\boldsymbol{\varphi}_{n}=\left(\varphi_{n}^{x}, \varphi_{n}^{y}\right)^{t}$ and $\boldsymbol{\Delta} \boldsymbol{u}_{n}=\left(\Delta^{x} u_{n}^{x}, \Delta^{y} u_{n}^{y}\right)^{t}$. Then:

$$
\int_{\Omega} \boldsymbol{\Delta} \boldsymbol{u}_{n} \cdot \boldsymbol{\varphi}_{n} \mathrm{~d} \boldsymbol{x}+\int_{\Omega} \boldsymbol{\nabla}_{\mathcal{M}} p_{n} \cdot \boldsymbol{\varphi}_{n} \mathrm{~d} \boldsymbol{x}=\int_{\Omega} \boldsymbol{f}_{n} \cdot \boldsymbol{\varphi}_{n} \mathrm{~d} \boldsymbol{x} .
$$

The convergence of the first term may be proven by slight modifications of a classical result $[4$, Chapter III]:

$$
\lim _{n \rightarrow \infty} \int_{\Omega} \boldsymbol{\Delta} \boldsymbol{u}_{n} \cdot \boldsymbol{\varphi}_{n} \mathrm{~d} \boldsymbol{x}=\int_{\Omega} \boldsymbol{\nabla} \boldsymbol{u} \cdot \boldsymbol{\nabla} \boldsymbol{\varphi} \mathrm{d} \boldsymbol{x} .
$$

From the definition of $\varphi_{n}$ and thanks to the weak convergence of the pressure, we have:

$\int_{\Omega} \boldsymbol{\nabla}_{\mathcal{M}} p_{n} \cdot \boldsymbol{\varphi}_{n} \mathrm{~d} \boldsymbol{x}=\int_{\Omega} p_{n} \operatorname{div} \boldsymbol{\varphi} \mathrm{d} \boldsymbol{x}$ and therefore $\lim _{n \rightarrow \infty} \int_{\Omega} \boldsymbol{\nabla}_{\mathcal{M}} p_{n} \cdot \boldsymbol{\varphi}_{n} \mathrm{~d} \boldsymbol{x}=\int_{\Omega} p \operatorname{div} \boldsymbol{\varphi} \mathrm{d} \boldsymbol{x}$.

Finally, since $\lim _{n \rightarrow \infty} \int_{\Omega} \boldsymbol{f}_{n} \cdot \boldsymbol{\varphi}_{n} \mathrm{~d} \boldsymbol{x}=\int_{\Omega} \boldsymbol{f} \cdot \boldsymbol{\varphi} \mathrm{d} \boldsymbol{x}$, the functions $\boldsymbol{u}$ and $p$ satisfy (2.2a).

Now let $\psi \in \mathrm{C}_{c}^{\infty}(\Omega)$, and let $\psi_{n} \in S_{n}$ with values $\left(\psi_{n}\right)_{i, j}=\psi\left(x_{i}, y_{j}\right)$ on $K_{i, j}$, for $1 \leq i \leq N, 1 \leq j \leq M$, i.e. $\left(\psi_{n}\right)_{i, j}$ is the midpoint value of $\psi$ in the respective box $(i, j)$ of the mesh. Note that, in this relation and in the remainder of the proof, the dependency of the mesh on $n$ has been omitted for short. Let us multiply the discrete 
mass balance equation (4.2c) corresponding to $K_{i, j}$ by $h_{i}^{x} h_{j}^{y}\left(\psi_{n}\right)_{i, j}$ and sum over $i$ and $j$, to obtain $T_{s, n}+T_{x, n}+T_{y, n}=0$, with:

$$
\begin{aligned}
T_{s, n} & =h_{n}^{\alpha} \sum_{i=1}^{N} \sum_{j=1}^{M} h_{i}^{x} h_{j}^{y}\left(\psi_{n}\right)_{i, j}\left[\left(\rho_{n}\right)_{i, j}-\rho^{*}\right], \\
T_{x, n} & =\sum_{i=1}^{N} \sum_{j=1}^{M} h_{j}^{y}\left(\psi_{n}\right)_{i, j}\left[\left(\rho_{n}\right)_{i+\frac{1}{2}, j}\left(u_{n}^{x}\right)_{i+\frac{1}{2}, j}-\left(\rho_{n}\right)_{i-\frac{1}{2}, j}\left(u_{n}^{x}\right)_{i-\frac{1}{2}, j}\right], \\
T_{y, n} & =\sum_{i=1}^{N} \sum_{j=1}^{M} h_{i}^{x}\left(\psi_{n}\right)_{i, j}\left[\left(\rho_{n}\right)_{i, j+\frac{1}{2}}\left(u_{n}^{y}\right)_{i, j+\frac{1}{2}}-\left(\rho_{n}\right)_{i, j-\frac{1}{2}}\left(u_{n}^{y}\right)_{i, j-\frac{1}{2}}\right] .
\end{aligned}
$$

Since the sequence $\left(\rho_{n}\right)_{n \in \mathbb{N}}$ is bounded in $\mathrm{L}^{1}(\Omega)$, the term $T_{s, n}$ tends to zero for any $\alpha>0$. Reordering the summations, we get for $T_{x, n}$ :

$$
T_{x, n}=\sum_{i=1}^{N} \sum_{j=1}^{M}-h_{i+\frac{1}{2}}^{x} h_{j}^{y}\left(\rho_{n}\right)_{i+\frac{1}{2}, j}\left(u_{n}^{x}\right)_{i+\frac{1}{2}, j} \frac{\left(\psi_{n}\right)_{i+1, j}-\left(\psi_{n}\right)_{i, j}}{h_{i+\frac{1}{2}}^{x}} .
$$

Since we have:

$$
\left|\frac{\left(\psi_{n}\right)_{i+1, j}-\left(\psi_{n}\right)_{i, j}}{h_{i+\frac{1}{2}}^{x}}-\frac{1}{h_{i+\frac{1}{2}}^{x} h_{j}^{y}} \int_{K_{i+\frac{1}{2}, j}^{x}} \partial_{x} \psi \mathrm{d} \boldsymbol{x}\right| \leq c_{\psi} h_{n}
$$

where $\partial_{x}$ denotes the partial derivative with respect to the $x$ variable and $c_{\psi} \in \mathbb{R}$ depends only on $\psi$, we get $T_{x, n}=T_{1, n}+R_{1, n}$ with:

$$
T_{1, n}=\sum_{i=1}^{N} \sum_{j=1}^{M} \int_{K_{i+\frac{1}{2}, j}^{x}}\left(\rho_{n}\right)_{i+\frac{1}{2}, j}\left(u_{n}^{x}\right)_{i+\frac{1}{2}, j} \partial_{x} \psi \mathrm{d} \boldsymbol{x},
$$

and, by the Cauchy-Schwarz inequality and thanks to (3.2):

$$
\begin{aligned}
\left|R_{1, n}\right| & \leq c_{\psi} h_{n} \sum_{i=1}^{N} \sum_{j=1}^{M} h_{i+\frac{1}{2}}^{x} h_{j}^{y}\left|\left(\rho_{n}\right)_{i+\frac{1}{2}, j}\left(u_{n}^{x}\right)_{i+\frac{1}{2}, j}\right| \\
& \leq c h_{n}\left\|\rho_{n}\right\|_{\mathrm{L}^{2}(\Omega)}\left\|u_{n}^{x}\right\|_{\mathrm{L}^{2}(\Omega)},
\end{aligned}
$$

where $c$ depends only on $M, \Omega$ and $\boldsymbol{f}$ through the bounds on $\rho_{n}$ and $u_{n}$ (see Proposition 5.5) and on $\psi$ and the parameter $\eta$ defined in (3.2). Therefore $R_{1, n}$ tends to zero when $n \rightarrow \infty$. Now we can decompose $T_{1, n}=T_{2, n}+R_{2, n}$ with:

$$
T_{2, n}=\int_{\Omega} \rho_{n} u_{n}^{x} \partial_{x} \psi \mathrm{d} \boldsymbol{x}, \quad R_{2, n}=\sum_{i=1}^{N} \sum_{j=1}^{M} \int_{K_{i+\frac{1}{2}, j}^{x}}\left[\left(\rho_{n}\right)_{i+\frac{1}{2}, j}-\rho_{n}\right]\left(u_{n}^{x}\right)_{i+\frac{1}{2}, j} \partial_{x} \psi \mathrm{d} \boldsymbol{x} .
$$

On one hand, by definition, the quantity $\left(\rho_{n}\right)_{i+\frac{1}{2}, j}$ is equal either to $\left(\rho_{n}\right)_{i, j}$ or to $\left(\rho_{n}\right)_{i+1, j}$. On the other hand, over $K_{i+\frac{1}{2}, j}^{x}, \rho_{n}$ is equal to $\left(\rho_{n}\right)_{i, j}$ on the " $i-$ side of the edge" and to $\left(\rho_{n}\right)_{i+1, j}$ on the " $(i+1)$ - side". Since $\partial_{x} \psi$ is bounded, we thus get:

$$
\left|R_{2, n}\right| \leq c_{\psi} \sum_{i=1}^{N} \sum_{j=1}^{M} h_{i+\frac{1}{2}}^{x} h_{j}^{y}\left|\left(u_{n}^{x}\right)_{i+\frac{1}{2}, j}\right|\left|\left(\rho_{n}\right)_{i+1, j}-\left(\rho_{n}\right)_{i, j}\right| .
$$


Let us first suppose that $\gamma \geq 2$. We have:

$$
\begin{aligned}
\left|R_{2, n}\right| \leq c_{\psi} h_{n}^{1 / 2}\left[\sum_{i=1}^{N} \sum_{j=1}^{M} h_{j}^{y}\left|\left(u_{n}^{x}\right)_{i+\frac{1}{2}, j}\right|\right. & \left.\left|\left(\rho_{n}\right)_{i+1, j}-\left(\rho_{n}\right)_{i, j}\right|^{2}\right]^{1 / 2} \\
& {\left[\sum_{i=1}^{N} \sum_{j=1}^{M} h_{i+\frac{1}{2}}^{x} h_{j}^{y}\left|\left(u_{n}^{x}\right)_{i+\frac{1}{2}, j}\right|\right]^{1 / 2} . }
\end{aligned}
$$

The last two quantities are controlled, by the second inequality of Proposition 5.5, i.e. Relation (5.7), for the first one and because $\left(u_{n}^{x}\right)_{n \in \mathbb{N}}$ is bounded in $\mathrm{L}^{1}(\Omega)$ for the second one. So we get $\left|R_{2, n}\right| \leq c h_{n}^{1 / 2}$ with $c$ independent of $n$.

If we now suppose that $\gamma<2$, we may write:

$$
\begin{aligned}
\left|R_{2, n}\right| \leq c_{\psi} h_{n}^{1 / 2}\left[\sum_{i=1}^{N} \sum_{j=1}^{M} h_{j}^{y}\left(\left(\tilde{\rho}_{n}\right)_{i+\frac{1}{2}, j}\right)^{\gamma-2}\left|\left(u_{n}^{x}\right)_{i+\frac{1}{2}, j}\right|\left|\left(\rho_{n}\right)_{i+1, j}-\left(\rho_{n}\right)_{i, j}\right|^{2}\right]^{1 / 2} \\
\\
{\left[\sum_{i=1}^{N} \sum_{j=1}^{M} h_{i+\frac{1}{2}}^{x} h_{j}^{y}\left(\left(\tilde{\rho}_{n}\right)_{i+\frac{1}{2}, j}\right)^{2-\gamma}\left|\left(u_{n}^{x}\right)_{i+\frac{1}{2}, j}\right|\right]^{1 / 2} }
\end{aligned}
$$

where $\left(\tilde{\rho}_{n}\right)_{i+\frac{1}{2}, j}=\max \left(\left(\rho_{n}\right)_{i, j},\left(\rho_{n}\right)_{i+1, j}\right)$. Hence by $(5.7)$, the first sum is bounded. For the second one, we have:

$$
\begin{aligned}
\sum_{i=1}^{N} \sum_{j=1}^{M} h_{i+\frac{1}{2}}^{x} h_{j}^{y}\left(\left(\tilde{\rho}_{n}\right)_{i+\frac{1}{2}, j}\right)^{2-\gamma} & \left|\left(u_{n}^{x}\right)_{i+\frac{1}{2}, j}\right| \leq\left[\sum_{i=1}^{N} \sum_{j=1}^{M} h_{i+\frac{1}{2}}^{x} h_{j}^{y}\left(u_{n}^{x}\right)_{i+\frac{1}{2}, j}^{2}\right]^{1 / 2} \\
& {\left[\sum_{i=1}^{N} \sum_{j=1}^{M} h_{i+\frac{1}{2}}^{x} h_{j}^{y} \max \left(\left(\rho_{n}\right)_{i, j},\left(\rho_{n}\right)_{i+1, j}, 1\right)^{2}\right]^{1 / 2} }
\end{aligned}
$$

The first term in the right hand side is controlled since $\left\|u_{n}^{x}\right\|_{\mathrm{L}^{2}(\Omega)}$ is bounded and, the second one is controlled since $\left\|\rho_{n}\right\|_{\mathrm{L}^{2}(\Omega)}$ is bounded, using the regularity bound (3.2) on $h_{i+1}^{x} / h_{i}^{x}$. In this case also, $\left|R_{2, n}\right|$ tends to zero.

By the weak and strong convergence of $\left(\rho_{n}\right)_{n \in \mathbb{N}}$ and $\left(u_{n}^{x}\right)_{n \in \mathbb{N}}$ respectively, we have:

$$
\lim _{n \rightarrow \infty} T_{x, n}=\lim _{n \rightarrow \infty} T_{2, n}=\int_{\Omega} \rho u^{x} \partial_{x} \psi \mathrm{d} \boldsymbol{x} .
$$

By the same arguments, we obtain:

$$
\lim _{n \rightarrow \infty} T_{y, n}=\int_{\Omega} \rho u^{y} \partial_{y} \psi \mathrm{d} \boldsymbol{x},
$$

which shows that $\rho$ and $\boldsymbol{u}$ satisfy the continuous mass balance equation.

REMARK 6.1. Note that, in the case $\gamma \geq 2$, the convergence proof may easily be modified so as not to require that all the meshes $\mathcal{M}_{n}$ satisfy (3.2) with the same $\eta$ (it is used here only for short in order to get (6.1)). However, in the case $\gamma<2$, it does not seem easy to proceed without this regularity assumption, which is used to obtain (6.2). Moreover, the same assumption is also used in the proof of convergence of the equation of state, for any $\gamma$ (see Step 2 of Lemma 7.3). 
7. Passing to the limit in the equation of state. Under the hypotheses of Proposition 6.1, up to a subsequence, the approximate solution $\left(\boldsymbol{u}_{n}, p_{n}, \rho_{n}\right)$ converges (in the sense given in Proposition 6.1) to $(\boldsymbol{u}, p, \rho)$ (as $n \rightarrow \infty)$; the limit $(\boldsymbol{u}, p, \rho)$ belongs to $\mathrm{H}_{0}^{1}(\Omega)^{2} \times \mathrm{L}^{2}(\Omega) \times \mathrm{L}^{2 \gamma}(\Omega)$ and satisfies (2.2a), (2.2b), $\rho \geq 0$ a.e. and $\int_{\Omega} \rho \mathrm{d} \boldsymbol{x}=M$. There remains to show that $(\boldsymbol{u}, p, \rho)$ satisfies (2.1c), i.e. the equation of state $p=\rho^{\gamma}$ a.e. (and that the convergence of $p_{n}$ and $\rho_{n}$ are strong in the appropriate Lebesgue spaces).

If $\gamma=1$, this is easily proved (except for the strong convergences of $p_{n}$ and $\rho_{n}$ ), thanks to the weak convergence as $n \rightarrow \infty$ of $p_{n}$ and $\rho_{n}$ to $p$ and $\rho$ in $\mathrm{L}^{2}(\Omega)$. For $\gamma>1$ however, we may not pass to the limit in the nonlinear equality $p_{n}=\rho_{n}^{\gamma}$ since the convergence of $p_{n}$ towards $p$ and of $\rho_{n}$ towards $\rho$ are only weak in $\mathrm{L}^{2}(\Omega)$. In order to obtain the relation $p=\rho^{\gamma}$, the main idea is to prove that:

$$
\liminf _{n \rightarrow \infty} \int_{\Omega} p_{n} \rho_{n} \mathrm{~d} \boldsymbol{x} \leq \int_{\Omega} p \rho \mathrm{d} \boldsymbol{x} .
$$

Once this inequality is proven, it is quite easy to deduce (up to a subsequence) the a.e. convergence of $p_{n}$ and $\rho_{n}$ and therefore $p=\rho^{\gamma}$ (and we also obtain the desired strong convergence of $p_{n}$ and $\rho_{n}$ ).

7.1. Proof of (7.1) in a continuous setting. For the sake of clarity, we first prove (7.1) in a simple (but unrealistic) setting, namely assuming that $\boldsymbol{u}_{n}$ satisfies the following approximate (but not discrete) equation:

$$
\boldsymbol{u}_{n} \in \mathrm{H}_{0}^{1}(\Omega)^{2}, \quad-\Delta \boldsymbol{u}_{n}+\nabla p_{n}=\boldsymbol{f}_{n},
$$

with $\boldsymbol{f}_{n} \in \mathrm{L}^{2}(\Omega)^{2}$ and $\boldsymbol{f}_{n} \rightarrow \boldsymbol{f}$ weakly in $\mathrm{L}^{2}(\Omega)^{2}$ as $n \rightarrow \infty$. The proof we give here is slightly different from that of [5]. It is more adapted to the case of the MAC scheme that we consider here but it could also be used for the proof of convergence of the scheme considered in [5].

For all $\boldsymbol{v}, \boldsymbol{w}$ in $\mathrm{H}_{0}^{1}(\Omega)^{2}$ (in fact $\boldsymbol{w} \in \mathrm{H}^{1}(\Omega)^{2}$ is sufficient), one has:

$$
\int_{\Omega} \boldsymbol{\nabla} \boldsymbol{v}: \boldsymbol{\nabla} \boldsymbol{w} \mathrm{d} \boldsymbol{x}=\int_{\Omega} \operatorname{div} \boldsymbol{v} \operatorname{div} \boldsymbol{w} \mathrm{d} \boldsymbol{x}+\int_{\Omega} \operatorname{curl} \boldsymbol{v} \operatorname{curl} \boldsymbol{w} \mathrm{d} \boldsymbol{x} .
$$

with, for $\boldsymbol{v}=\left(v^{x}, v^{y}\right), \operatorname{curl}(\boldsymbol{v})=\partial_{x} v^{y}-\partial_{y} v^{x}\left(\partial_{x}\left(\partial_{y}\right)\right.$ denoting the derivative with respect to the $x$ (resp. $y$ ) space variable). Even though the relation (7.3) is wellknown, we now give its proof, which will later be used as a guide for the proof of the discrete counterpart of (7.3), stated in Lemma 7.1. Let $\boldsymbol{v}=\left(v^{x}, v^{y}\right) \in \mathrm{C}_{c}^{\infty}(\Omega)^{2}$, $\boldsymbol{w}=\left(w^{x}, w^{y}\right) \in \mathrm{C}_{c}^{\infty}(\Omega)^{2}$. A blunt comparison of the two sides of (7.3) using the definition of the differential operators yields:

$$
\int_{\Omega} \boldsymbol{\nabla} \boldsymbol{v}: \boldsymbol{\nabla} \boldsymbol{w} \mathrm{d} \boldsymbol{x}=\int_{\Omega} \operatorname{div} \boldsymbol{v} \operatorname{div} \boldsymbol{w} \mathrm{d} \boldsymbol{x}+\int_{\Omega} \operatorname{curl} \boldsymbol{v} \operatorname{curl} \boldsymbol{w} \mathrm{d} \boldsymbol{x}-R,
$$

with $R=\int_{\Omega} \partial_{x} v^{x} \partial_{y} w^{y}+\partial_{y} v^{y} \partial_{x} w^{x}-\partial_{y} v^{x} \partial_{x} w^{y}-\partial_{x} v^{y} \partial_{y} w^{x} \mathrm{~d} \boldsymbol{x}$.

A double integration by parts gives:

$$
\int_{\Omega} \partial_{x} v^{x} \partial_{y} w^{y} \mathrm{~d} \boldsymbol{x}=\int_{\Omega} \partial_{y} v^{x} \partial_{x} w^{y} \mathrm{~d} \boldsymbol{x} \quad \text { and } \quad \int_{\Omega} \partial_{y} v^{y} \partial_{x} w^{x} \mathrm{~d} \boldsymbol{x}=\int_{\Omega} \partial_{x} v^{y} \partial_{y} w^{x} \mathrm{~d} \boldsymbol{x},
$$

which leads to $R=0$ and (7.3). Then, we obtain (7.3) in $\mathrm{H}_{0}^{1}(\Omega)^{2}$ by density. 
REMARK 7.1 (Extension of (7.3) to the three-dimensional case). In the three dimensional case, the curl operator is defined by $\operatorname{curl} \boldsymbol{v}=\left(\partial_{y} v^{z}-\partial_{z} v^{y}, \partial_{z} v^{x}-\right.$ $\left.\partial_{x} v^{z}, \partial_{x} v^{y}-\partial_{y} v^{x}\right)^{t}$. The quantities $\operatorname{curl} \boldsymbol{v}$ and $\operatorname{curl} \boldsymbol{w}$ are thus vector valued but Relation (7.3) still holds, replacing the product $\operatorname{curl} \boldsymbol{v} \operatorname{curl} \boldsymbol{w}$ by the dot product $\operatorname{curl} \boldsymbol{v}$. $\operatorname{curl} \boldsymbol{w}$ in $\mathbb{R}^{3}$. The proof of (7.3) then follows by using three times the formula (7.3) written in the two dimensional case. More precisely, if $\boldsymbol{v}=\left(v^{x}, v^{y}, v^{z}\right) \in \mathrm{C}_{c}^{\infty}(\Omega)^{3}$, $\boldsymbol{w}=\left(w^{x}, w^{y}, w^{z}\right) \in \mathrm{C}_{c}^{\infty}(\Omega)^{3}$, one has, for $\left(\alpha_{1}, \alpha_{2}, \alpha_{3}\right) \in\{(x, y, z),(y, z, x),(z, x, y)\}$,

$$
\begin{aligned}
\int_{\Omega} \partial_{\alpha_{1}} v^{\alpha_{1}} \partial_{\alpha_{1}} w^{\alpha_{1}}+\partial_{\alpha_{2}} v^{\alpha_{1}} \partial_{\alpha_{2}} w^{\alpha_{1}}+\partial_{\alpha_{1}} v^{\alpha_{2}} \partial_{\alpha_{1}} w^{\alpha_{2}}+\partial_{\alpha_{2}} v^{\alpha_{2}} \partial_{\alpha_{2}} w^{\alpha_{2}} \mathrm{~d} \boldsymbol{x} \\
=\int_{\Omega}\left(\partial_{\alpha_{1}} v^{\alpha_{1}}+\partial_{\alpha_{2}} v^{\alpha_{2}}\right)\left(\partial_{\alpha_{1}} w^{\alpha_{1}}+\partial_{\alpha_{2}} w^{\alpha_{2}}\right) \mathrm{d} \boldsymbol{x}+\int_{\Omega} \operatorname{curl}^{\alpha_{3}} \boldsymbol{v} \operatorname{curl}^{\alpha_{3}} \boldsymbol{w} \mathrm{d} \boldsymbol{x},
\end{aligned}
$$

where $\operatorname{curl}^{\alpha_{3}}$ denotes the $\alpha_{3}$-th component of curl. Then, adding these three equations leads to:

$$
\begin{aligned}
& \int_{\Omega}\left[\boldsymbol{\nabla} \boldsymbol{v}: \boldsymbol{\nabla} \boldsymbol{w}+\sum_{\alpha \in\{x, y, z\}} \partial_{\alpha} v^{\alpha} \partial_{\alpha} w^{\alpha}\right] \mathrm{d} \boldsymbol{x}= \\
& \int_{\Omega}\left[\operatorname{div} \boldsymbol{v} \operatorname{div} \boldsymbol{w}+\sum_{\alpha \in\{x, y, z\}} \partial_{\alpha} v^{\alpha} \partial_{\alpha} w^{\alpha}+\operatorname{curl} \boldsymbol{v} \cdot \operatorname{curl} \boldsymbol{w}\right] \mathrm{d} \boldsymbol{x} .
\end{aligned}
$$

This gives (7.3) for $\boldsymbol{v}, \boldsymbol{w} \in \mathrm{C}_{c}^{\infty}(\Omega)^{3}$ and, by density, for $\boldsymbol{v}, \boldsymbol{w} \in \mathrm{H}_{0}^{1}(\Omega)^{3}$.

Using formula (7.3), we get from (7.2), for all $\boldsymbol{v}$ in $\mathrm{H}_{0}^{1}(\Omega)^{2}$,

$$
\int_{\Omega} \operatorname{div} \boldsymbol{u}_{n} \operatorname{div} \boldsymbol{v} \mathrm{d} \boldsymbol{x}+\int_{\Omega} \operatorname{curl} \boldsymbol{u}_{n} \operatorname{curl} \boldsymbol{v} \mathrm{d} \boldsymbol{x}-\int_{\Omega} p_{n} \operatorname{div} \boldsymbol{v} \mathrm{d} \boldsymbol{x}=\int_{\Omega} \boldsymbol{f}_{n} \cdot \boldsymbol{v} \mathrm{d} \boldsymbol{x} .
$$

Let us assume, for a moment, that we may choose $\boldsymbol{v}=\boldsymbol{v}_{n}$ with:

$$
\operatorname{curl} \boldsymbol{v}_{n}=0, \quad \operatorname{div} \boldsymbol{v}_{n}=\rho_{n},
$$

and $\boldsymbol{v}_{n}$ bounded in $\mathrm{H}_{0}^{1}(\Omega)^{2}$. Unfortunately, such a choice is possible in $\mathrm{H}^{1}(\Omega)^{2}$ but not in $\mathrm{H}_{0}^{1}(\Omega)^{2}$, and we shall see later how to get around this difficulty. With such a choice of $\boldsymbol{v}_{n}$, we have up to a subsequence, $\boldsymbol{v}_{n} \rightarrow \boldsymbol{v}$ in $\mathrm{L}^{2}(\Omega)^{2}$ and weakly in $\mathrm{H}_{0}^{1}(\Omega)^{2}$ as $n \rightarrow \infty$ with:

$$
\operatorname{curl} \boldsymbol{v}=0, \operatorname{div} \boldsymbol{v}=\rho .
$$

Since $\operatorname{div} \boldsymbol{v}_{n}=\rho_{n}$ and curl $\boldsymbol{v}_{n}=0,(7.4) \operatorname{reads} \int_{\Omega}\left(\operatorname{div} \boldsymbol{u}_{n}-p_{n}\right) \rho_{n} \mathrm{~d} \boldsymbol{x}=\int_{\Omega} \boldsymbol{f}_{n} \cdot \boldsymbol{v}_{n} \mathrm{~d} \boldsymbol{x}$. Since $\boldsymbol{f}_{n}$ and $\boldsymbol{v}_{n}$ weakly converge in $\mathrm{L}^{2}(\Omega)^{2}$ to $\boldsymbol{f}$ and $\boldsymbol{v}$ respectively, we obtain:

$$
\lim _{n \rightarrow \infty} \int_{\Omega}\left(\operatorname{div} \boldsymbol{u}_{n}-p_{n}\right) \rho_{n} \mathrm{~d} \boldsymbol{x}=\int_{\Omega} \boldsymbol{f} \cdot \boldsymbol{v} \mathrm{d} \boldsymbol{x} .
$$

But, since $(\boldsymbol{u}, p)$ satisfies $(2.2 \mathrm{a})$, one has:

$$
\int_{\Omega} \operatorname{div} \boldsymbol{u} \operatorname{div} \boldsymbol{v} \mathrm{d} \boldsymbol{x}+\int_{\Omega} \operatorname{curl} \boldsymbol{u} \operatorname{curl} \boldsymbol{v} \mathrm{d} \boldsymbol{x}-\int_{\Omega} p \operatorname{div} \boldsymbol{v} \mathrm{d} \boldsymbol{x}=\int_{\Omega} \boldsymbol{f} \cdot \boldsymbol{v} \mathrm{d} \boldsymbol{x} .
$$

which gives, thanks to (7.6), $\int_{\Omega}(\operatorname{div} \boldsymbol{u}-p) \rho \mathrm{d} \boldsymbol{x}=\int_{\Omega} \boldsymbol{f} \cdot \boldsymbol{v} \mathrm{d} \boldsymbol{x}$. Therefore:

$$
\lim _{n \rightarrow \infty} \int_{\Omega}\left(p_{n}-\operatorname{div} \boldsymbol{u}_{n}\right) \rho_{n} \mathrm{~d} \boldsymbol{x}=\int_{\Omega}(p-\operatorname{div} \boldsymbol{u}) \rho \mathrm{d} \boldsymbol{x} .
$$


Finally, thanks to the mass balance equation (2.2b), Lemma 2.1 of [5] gives:

$$
\int_{\Omega} \rho \operatorname{div} \boldsymbol{u} \mathrm{d} \boldsymbol{x}=0
$$

We assume also that $\boldsymbol{u}_{n}$ satisfies an approximate mass balance equation leading to:

$$
\liminf _{n \rightarrow \infty} \int_{\Omega} \rho_{n} \operatorname{div} \boldsymbol{u}_{n} \mathrm{~d} \boldsymbol{x} \leq 0 .
$$

This holds in the discrete case, that is when $\rho_{n}$ and $\boldsymbol{u}_{n}$ satisfy (4.2c), as stated in Lemma 5.4. Then one deduces (7.1) from (7.7).

Unfortunately, the above proof of (7.1) fails because it is impossible to choose $\boldsymbol{v}_{n}$ in $\mathrm{H}_{0}^{1}(\Omega)^{2}$. In order to overcome this, we implement the following construction for $\boldsymbol{v}_{n}$, which we sketch formally, since it gives the building blocks for a similar construction in the discrete MAC setting in the following subsection. We first choose $w_{n}$ such that $w_{n} \in \mathrm{H}_{0}^{1}(\Omega)$ and $-\Delta w_{n}=\rho_{n}$. Since the sequence $\left(\rho_{n}\right)_{n \in \mathbb{N}}$ is bounded in $L^{2}(\Omega)$, the sequence $\left(w_{n}\right)_{n \in \mathbb{N}}$ is bounded in $H_{0}^{1}(\Omega)$. But we can then also derive an $\mathrm{H}_{l o c}^{2}(\Omega)$ estimate on $w_{n}$ (an $\mathrm{H}^{2}(\Omega)$-estimate is also available if $\Omega$ is convex; however this assumption is otherwise not needed in the present paper); indeed, for $\varphi \in \mathrm{C}_{c}^{\infty}(\Omega)$ the sequence $\left(\Delta\left(w_{n} \varphi\right)\right)_{n \in \mathbb{N}}$ is bounded in $\mathrm{L}^{2}(\Omega)$ (by some quantity depending on $\varphi$ ), two "formal" integrations by parts (which can be justified) give:

$$
\begin{gathered}
\sum_{\alpha_{1}, \alpha_{2} \in\{x, y\}} \int_{\Omega} \partial_{\alpha_{1}} \partial_{\alpha_{2}}\left(w_{n} \varphi\right) \partial_{\alpha_{1}} \partial_{\alpha_{2}}\left(w_{n} \varphi\right) \mathrm{d} \boldsymbol{x}= \\
\sum_{\alpha_{1}, \alpha_{2} \in\{x, y\}} \int_{\Omega} \partial_{\alpha_{1}} \partial_{\alpha_{1}}\left(w_{n} \varphi\right) \partial_{\alpha_{2}} \partial_{\alpha_{2}}\left(w_{n} \varphi\right) \mathrm{d} \boldsymbol{x}=\int_{\Omega}\left(\Delta\left(w_{n} \varphi\right)\right)^{2} \mathrm{~d} \boldsymbol{x} .
\end{gathered}
$$

Since $\left(w_{n}\right)_{n \in \mathbb{N}}$ is bounded in $H_{0}^{1}(\Omega)$ and in $\mathrm{H}_{l o c}^{2}(\Omega)$, up to a subsequence, $w_{n} \rightarrow w$ weakly in $H_{0}^{1}(\Omega)$ and in $\mathrm{H}_{l o c}^{2}(\Omega)$ as $n \rightarrow \infty$, so that $w_{n} \rightarrow w$ in $L^{2}(\Omega)$ and $\boldsymbol{\nabla}\left(w_{n} \varphi\right) \rightarrow$ $\boldsymbol{\nabla}(w \varphi)$ weakly in $H_{0}^{1}(\Omega)^{2}$ and (strongly) in $L^{2}(\Omega)^{2}$ for all $\varphi \in C_{c}^{\infty}(\Omega)$. Setting $\boldsymbol{v}_{n}=-\boldsymbol{\nabla} w_{n}$ and $\boldsymbol{v}=-\boldsymbol{\nabla} w$, we then have $\boldsymbol{v}_{n} \in \mathrm{H}_{l o c}^{1}(\Omega)^{2}$, $\operatorname{div} \boldsymbol{v}_{n}=\rho_{n}$ a.e. in $\Omega$, curl $\boldsymbol{v}_{n}=0$ a.e. in $\Omega$ and, as $n \rightarrow \infty, \boldsymbol{v}_{n} \rightarrow \boldsymbol{v}$ in $\mathrm{L}_{l o c}^{2}(\Omega)^{2}$ and weakly in $\mathrm{H}_{l o c}^{1}(\Omega)^{2}$, with (7.6) (i.e. $\operatorname{curl} \boldsymbol{v}=0, \operatorname{div} \boldsymbol{v}=\rho$ ).

Let $\varphi \in \mathrm{C}_{c}^{\infty}(\Omega)$, so that $\boldsymbol{v}_{n} \varphi \in \mathrm{H}_{0}^{1}(\Omega)^{2}$. Let us now prove that:

$$
\lim _{n \rightarrow \infty} \int_{\Omega}\left(p_{n}-\operatorname{div} \boldsymbol{u}_{n}\right) \rho_{n} \varphi \mathrm{d} \boldsymbol{x}=\int_{\Omega}(p-\operatorname{div} \boldsymbol{u}) \rho \varphi \mathrm{d} \boldsymbol{x} .
$$

Taking $\boldsymbol{v}=\boldsymbol{v}_{n} \varphi,(7.4)$ gives:

$$
\begin{aligned}
\int_{\Omega} \operatorname{div} \boldsymbol{u}_{n} \operatorname{div}\left(\boldsymbol{v}_{n} \varphi\right) \mathrm{d} \boldsymbol{x}+\int_{\Omega} \operatorname{curl} \boldsymbol{u}_{n} \operatorname{curl}\left(\boldsymbol{v}_{n} \varphi\right) \mathrm{d} \boldsymbol{x} & \\
& -\int_{\Omega} p_{n} \operatorname{div}\left(\boldsymbol{v}_{n} \varphi\right) \mathrm{d} \boldsymbol{x}=\int_{\Omega} \boldsymbol{f}_{n} \cdot\left(\boldsymbol{v}_{n} \varphi\right) \mathrm{d} \boldsymbol{x} .
\end{aligned}
$$

But, $\operatorname{div}\left(\boldsymbol{v}_{n} \varphi\right)=\rho_{n} \varphi+\boldsymbol{v}_{n} \cdot \boldsymbol{\nabla} \varphi$ and $\operatorname{curl}\left(\boldsymbol{v}_{n} \varphi\right)=L(\varphi) \boldsymbol{v}_{n}$, where $L(\varphi)$ is a $1 \times 2$-matrix (in the three dimensional case, a $3 \times 3$-matrix) involving the first order derivatives of $\varphi$. Thus:

$$
\begin{aligned}
\int_{\Omega}\left(\operatorname{div} \boldsymbol{u}_{n}\right. & \left.-p_{n}\right) \rho_{n} \varphi \mathrm{d} \boldsymbol{x}=\int_{\Omega} \boldsymbol{f}_{n} \cdot\left(\boldsymbol{v}_{n} \varphi\right) \mathrm{d} \boldsymbol{x} \\
& -\int_{\Omega}\left(\operatorname{div} \boldsymbol{u}_{n}\right) \boldsymbol{v}_{n} \cdot \nabla \varphi \mathrm{d} \boldsymbol{x}-\int \operatorname{curl} \boldsymbol{u}_{n} L(\varphi) \boldsymbol{v}_{n} \mathrm{~d} \boldsymbol{x}+\int_{\Omega} p_{n} \boldsymbol{v}_{n} \cdot \nabla \varphi \mathrm{d} \boldsymbol{x}
\end{aligned}
$$


Using the weak convergence of $\boldsymbol{u}_{n}$ in $\mathrm{H}_{0}^{1}(\Omega)^{2}$, the weak convergence of $p_{n}$ and $\boldsymbol{f}_{n}$ in $\mathrm{L}^{2}(\Omega)$ and $\mathrm{L}^{2}(\Omega)^{2}$ respectively and the convergence of $\boldsymbol{v}_{n}$ in $\mathrm{L}_{l o c}^{2}(\Omega)^{2}$, we obtain:

$$
\begin{aligned}
\lim _{n \rightarrow \infty} \int_{\Omega}\left(\operatorname{div} \boldsymbol{u}_{n}-p_{n}\right) \rho_{n} \varphi \mathrm{d} \boldsymbol{x}=\int_{\Omega} f \cdot(\boldsymbol{v} \varphi) \mathrm{d} \boldsymbol{x} \\
\quad-\int_{\Omega}(\operatorname{div} \boldsymbol{u}) \boldsymbol{v} \cdot \nabla \varphi \mathrm{d} \boldsymbol{x}-\int \operatorname{curl} \boldsymbol{u} L(\varphi) \boldsymbol{v} \mathrm{d} \boldsymbol{x}+\int_{\Omega} p \boldsymbol{v} \cdot \nabla \varphi \mathrm{d} \boldsymbol{x} .
\end{aligned}
$$

But, since $(\boldsymbol{u}, p)$ satisfies $(2.2 \mathrm{a})$,

$$
\int_{\Omega} \operatorname{div} \boldsymbol{u} \operatorname{div}(\boldsymbol{v} \varphi) \mathrm{d} \boldsymbol{x}+\int_{\Omega} \operatorname{curl} \boldsymbol{u} \operatorname{curl}(\boldsymbol{v} \varphi) \mathrm{d} \boldsymbol{x}-\int_{\Omega} p \operatorname{div}(\boldsymbol{v} \varphi) \mathrm{d} \boldsymbol{x}=\int_{\Omega} \boldsymbol{f} \cdot(\boldsymbol{v} \varphi) \mathrm{d} \boldsymbol{x},
$$

which yields, using (7.6):

$$
\begin{aligned}
\int_{\Omega}(\operatorname{div} \boldsymbol{u} & -p) \rho \varphi \mathrm{d} \boldsymbol{x}=\int_{\Omega} \boldsymbol{f} \cdot(\boldsymbol{v} \varphi) \mathrm{d} \boldsymbol{x} \\
& -\int_{\Omega}(\operatorname{div} \boldsymbol{u}) \boldsymbol{v} \cdot \boldsymbol{\nabla} \varphi \mathrm{d} \boldsymbol{x}-\int_{\Omega} \operatorname{curl} \boldsymbol{u} L(\varphi) \boldsymbol{v} \mathrm{d} \boldsymbol{x}+\int_{\Omega} p \boldsymbol{v} \cdot \boldsymbol{\nabla} \varphi \mathrm{d} \boldsymbol{x}
\end{aligned}
$$

which proves (7.9).

Using now the fact that $\left(p_{n}-\operatorname{div} \boldsymbol{u}_{n}\right) \rho_{n}$ is bounded in $\mathrm{L}^{q}(\Omega)$ for some $q>1$ (namely $q=2 \gamma /(\gamma+1)>1$, thanks to the $\mathrm{L}^{2}(\Omega)$ estimate on $\left(p_{n}-\operatorname{div} \boldsymbol{u}_{n}\right)$ and the $\mathrm{L}^{2 \gamma}(\Omega)$ estimate on $\rho_{n}$ ), we deduce from (7.9) (see Lemma B.2 of [5]) that:

$$
\lim _{n \rightarrow \infty} \int_{\Omega}\left(p_{n}-\operatorname{div} \boldsymbol{u}_{n}\right) \rho_{n} \mathrm{~d} \boldsymbol{x}=\int_{\Omega}(p-\operatorname{div} \boldsymbol{u}) \rho \mathrm{d} \boldsymbol{x} .
$$

The proof of (7.1) then folows from the mass equation (2.1b), as it was from (7.7).

7.2. Proof of (7.1) in the discrete MAC setting. We now have to prove (7.1) with $\left(\boldsymbol{u}_{n}, p_{n}\right)$ solution of the discrete momentum equation (4.3) rather than of the approximate continuous momentum equation (7.2). We use here the fact that the MAC scheme allows an identity similar to (7.3), given in Lemma 7.1 below. Note that this path is quite different from the Crouzeix-Raviart framework [5]; indeed, for this latter discretization, the analogue of (7.3) involves some jumps at the boundaries of the elements, and an ad hoc strategy (including the introduction in the scheme of a stabilization term) was necessary to circumvent this difficulty.

We first define, in the framework of Section 3, that is with a MAC grid denoted by $\mathcal{M}$, the discrete operator $\operatorname{curl}_{\mathcal{M}}$, using the fourth partition of $\Omega$ and the space $S^{x y}$ defined in Section 3. The operator $\operatorname{curl}_{\mathcal{M}}$ is defined from $S^{x} \times S^{y}$ to $S^{x y}$; for $\boldsymbol{v}=\left(v^{x}, v^{y}\right) \in S^{x} \times S^{y}$ and for $i \in\{1, \ldots, N+1\}, j \in\{1, \ldots, M+1\}$, one has:

$$
\operatorname{curl}_{\mathcal{M}} \boldsymbol{v}=\frac{v_{i, j-\frac{1}{2}}^{y}-v_{i-1, j-\frac{1}{2}}^{y}}{h_{i-\frac{1}{2}}^{x}}-\frac{v_{i-\frac{1}{2}, j}^{x}-v_{i-\frac{1}{2}, j-1}^{x}}{h_{j-\frac{1}{2}}^{y}} \text { on } K_{i-\frac{1}{2}, j-\frac{1}{2}},
$$

where $h_{j+\frac{1}{2}}^{x}$ and $h_{i+\frac{1}{2}}^{y}$ are defined by (3.3) and

$$
v_{i-\frac{1}{2}, 0}^{x}=0, v_{i-\frac{1}{2}, M+1}^{x}=0, v_{0, j-\frac{1}{2}}^{y}=0, v_{N+1, j-\frac{1}{2}}^{y}=0 .
$$

In fact, one has $\operatorname{curl}_{\mathcal{M}} \boldsymbol{v}=\partial_{\mathcal{M}}^{x} v^{y}-\partial_{\mathcal{M}}^{y} v^{x}$, where $\partial_{\mathcal{M}}^{x} v^{y}$ and $\partial_{\mathcal{M}}^{y} v^{x}$ are defined by (3.10). More precisely, in (3.10), the quantities $\partial_{\mathcal{M}}^{x} v^{y}$ and $\partial_{\mathcal{M}}^{y} v^{x}$ were defined for $\boldsymbol{v} \in S_{0}^{x} \times S_{0}^{y}$; they are extended here to the case of $\boldsymbol{v} \in S^{x} \times S^{y}$. 
Lemma 7.1. Let $\mathcal{M}$ be a MAC grid, $\boldsymbol{v}=\left(v^{x}, v^{y}\right) \in S_{0}^{x} \times S_{0}^{y}$ and $\boldsymbol{w}=\left(w^{x}, w^{y}\right) \in$ $S_{0}^{x} \times S_{0}^{y}$. Then the following discrete identity holds:

$$
\left(v^{x}, w^{x}\right)^{x}+\left(v^{y}, w^{y}\right)^{y}=\int_{\Omega} \operatorname{div}_{\mathcal{M}} \boldsymbol{v} \operatorname{div}_{\mathcal{M}} \boldsymbol{w} \mathrm{d} \boldsymbol{x}+\int_{\Omega} \operatorname{curl}_{\mathcal{M}} \boldsymbol{v} \operatorname{curl}_{\mathcal{M}} \boldsymbol{w} \mathrm{d} \boldsymbol{x} .
$$

Proof. As in the continuous case, a blunt comparison of the two sides of (7.11) using the definition of the discrete differential operators yields:

$$
\left(v^{x}, w^{x}\right)^{x}+\left(v^{y}, w^{y}\right)^{y}=\int_{\Omega} \operatorname{div}_{\mathcal{M}} \boldsymbol{v} \operatorname{div}_{\mathcal{M}} \boldsymbol{w}+\int_{\Omega} \operatorname{curl}_{\mathcal{M}} \boldsymbol{v} \operatorname{curl}_{\mathcal{M}} \boldsymbol{w}-R_{\mathcal{M}},
$$

where $R_{\mathcal{M}}$ is a discrete version of the quantity $R$ obtained in the continuous case, that is $R_{\mathcal{M}}=A+B-C-D$ with:

$$
\begin{aligned}
& B=\sum_{i=1}^{N} \sum_{j=1}^{M}\left(\frac{v_{i, j+\frac{1}{2}}^{y}-v_{i, j-\frac{1}{2}}^{y}}{h_{j}^{y}}\right)\left(\frac{w_{i+\frac{1}{2}, j}^{x}-w_{i-\frac{1}{2}, j}^{x}}{h_{i}^{x}}\right) h_{j}^{y} h_{i}^{x}, \\
& D=\sum_{i=1}^{N+1} \sum_{j=1}^{M+1}\left(\frac{v_{i, j-\frac{1}{2}}^{y}-v_{i-1, j-\frac{1}{2}}^{y}}{h_{i-\frac{1}{2}}^{x}}\right)\left(\frac{w_{i-\frac{1}{2}, j}^{x}-w_{i-\frac{1}{2}, j-1}^{x}}{h_{j-\frac{1}{2}}^{y}}\right) h_{i-\frac{1}{2}}^{x} h_{j-\frac{1}{2}}^{y} .
\end{aligned}
$$

The terms $A$ and $C$ are the same as $B$ and $D$, exchanging $i$ and $j, N$ and $M$ and $x$ and $y$. The terms $A$ and $B$ come from $\int_{\Omega} \operatorname{div}_{\mathcal{M}} \boldsymbol{v} \operatorname{div}_{\mathcal{M}} \boldsymbol{w} \mathrm{d} \boldsymbol{x}$ and the terms $C$ and $D$ come from $\int_{\Omega} \operatorname{curl}_{\mathcal{M}} \boldsymbol{v} \operatorname{curl}_{\mathcal{M}} \boldsymbol{w} \mathrm{d} \boldsymbol{x}$. In order to prove (7.11), it is sufficient to prove $B=D$ (and, similarly, $A=C$ ). To this purpose, we use a double discrete integration by parts:

$$
\begin{aligned}
D & =\sum_{i=1}^{N+1} \sum_{j=1}^{M+1}\left(v_{i, j-\frac{1}{2}}^{y}-v_{i-1, j-\frac{1}{2}}^{y}\right)\left(w_{i-\frac{1}{2}, j}^{x}-w_{i-\frac{1}{2}, j-1}^{x}\right) \\
& =\sum_{i=1}^{N+1} \sum_{j=1}^{M+1} v_{i, j-\frac{1}{2}}^{y}\left(w_{i-\frac{1}{2}, j}^{x}-w_{i-\frac{1}{2}, j-1}^{x}\right)-\sum_{i=0}^{N} \sum_{j=1}^{M+1} v_{i, j-\frac{1}{2}}^{y}\left(w_{i+\frac{1}{2}, j}^{x}-w_{i+\frac{1}{2}, j-1}^{x}\right) .
\end{aligned}
$$

By (3.11) (i.e. $v_{0, j-\frac{1}{2}}^{y}=v_{N+1, j-\frac{1}{2}}^{y}=0$ for all $j \in\{1, \ldots, M+1\}$ ), we get:

$$
\begin{aligned}
D & =\sum_{i=1}^{N} \sum_{j=1}^{M+1} v_{i, j-\frac{1}{2}}^{y}\left(w_{i-\frac{1}{2}, j}^{x}-w_{i-\frac{1}{2}, j-1}^{x}-w_{i+\frac{1}{2}, j}^{x}-w_{i+\frac{1}{2}, j-1}^{x}\right) \\
& =\sum_{i=1}^{N} \sum_{j=1}^{M+1} v_{i, j-\frac{1}{2}}^{y}\left(w_{i-\frac{1}{2}, j}^{x}-w_{i+\frac{1}{2}, j}^{x}\right)-\sum_{i=1}^{N} \sum_{j=0}^{M} v_{i, j+\frac{1}{2}}^{y}\left(w_{i-\frac{1}{2}, j}^{x}-w_{i+\frac{1}{2}, j}^{x}\right) .
\end{aligned}
$$

Since $v^{y} \in S_{0}^{y}$, one has $v_{i, \frac{1}{2}}^{y}=v_{i, M+\frac{1}{2}}^{y}=0$ for all $i \in\{1, \ldots, N\}$. Therefore:

$$
D=\sum_{i=1}^{N} \sum_{j=1}^{M}\left(v_{i, j-\frac{1}{2}}^{y}-v_{i, j+\frac{1}{2}}^{y}\right)\left(w_{i-\frac{1}{2}, j}^{x}-w_{i+\frac{1}{2}, j}^{x}\right)=B .
$$

REMARK 7.2 (Three-dimensional case). If $d=3$, the operator $\operatorname{curl}_{\mathcal{M}}$ is vector valued and, for instance, with natural notations, the third component of $\operatorname{curl}_{\mathcal{M}}$ is 
defined on a space denoted $S^{x y}$ which stands for piecewise constant functions over each $K_{i-\frac{1}{2}, j-\frac{1}{2}, k}$, with $K_{i-\frac{1}{2}, j-\frac{1}{2}, k}=\left(x_{i-1}, x_{i}\right) \times\left(y_{j-1}, y_{j}\right) \times\left(z_{k-\frac{1}{2}}, z_{k+\frac{1}{2}}\right)$ and the formula for this component of $\operatorname{curl}_{\mathcal{M}} \boldsymbol{v}$ is the same as in the case $d=2$, adding the subscript $k$.

The three-dimensional operator $\operatorname{curl}_{\mathcal{M}}$ also satisfies Lemma 7.1. As in the continuous case (see Remark 7.1), the proof of this result is obtained by using three times the proof of the two-dimensional case.

Let us now return to the setting described at the beginning of this section. Assuming that the hypotheses of Proposition 6.1 are satisfied, $\left(\boldsymbol{u}_{n}, p_{n}, \rho_{n}\right)$ converges (in the sense given in Proposition 6.1) to $(\boldsymbol{u}, p, \rho)($ as $n \rightarrow \infty)$ and the limit $(\boldsymbol{u}, p, \rho)$ belongs to $\mathrm{H}_{0}^{1}(\Omega)^{2} \times \mathrm{L}^{2}(\Omega) \times \mathrm{L}^{2 \gamma}(\Omega)$ and satisfies $(2.2 \mathrm{a}),(2.2 \mathrm{~b}), \rho \geq 0$ a.e. and $\int_{\Omega} \rho \mathrm{d} \boldsymbol{x}=M$. We want to prove that $p=\rho^{\gamma}$ a.e. (and that the convergence of $p_{n}$ and $\rho_{n}$ are strong in the appropriate Lebesgue spaces).

For simplicity, when we consider a sequence $\left(\mathcal{M}_{n}\right)_{n \in \mathbb{N}}$ of MAC grids, the index $\mathcal{M}_{n}$ is replaced by $n$ in the notation of discrete functions and operators, such as $\operatorname{div}_{\mathcal{M}}$, $\operatorname{curl}_{\mathcal{M}}$ or $\nabla_{\mathcal{M}}$. With these notations, since $\left(\boldsymbol{u}_{n}, p_{n}\right)$ satisfies $(4.2 \mathrm{a})$ and $(4.2 \mathrm{~b})$, one has for all $\boldsymbol{v} \in S_{0}^{x} \times S_{0}^{y}$, using (7.11):

$$
\int_{\Omega} \operatorname{div}_{n} \boldsymbol{u}_{n} \operatorname{div}_{n} \boldsymbol{v} \mathrm{d} \boldsymbol{x}+\int_{\Omega} \operatorname{curl}_{n} \boldsymbol{u}_{n} \operatorname{curl}_{n} \boldsymbol{v} \mathrm{d} \boldsymbol{x}-\int_{\Omega} p_{n} \operatorname{div}_{n} \boldsymbol{v} \mathrm{d} \boldsymbol{x}=\int_{\Omega} \boldsymbol{f}_{n} \cdot \boldsymbol{v} \mathrm{d} \boldsymbol{x}
$$

We now have to choose a suitable test function $\boldsymbol{v}$ in this relation. As in the simpler case described above, an optimal choice would be $\boldsymbol{v}=\boldsymbol{v}_{n} \in S_{0}^{x} \times S_{0}^{y}$ with $\operatorname{curl}_{n} \boldsymbol{v}_{n}=0$, $\operatorname{div}_{n} \boldsymbol{v}_{n}=\rho_{n}$ and $\boldsymbol{v}_{n}$ bounded for the natural norm of $S_{0}^{x} \times S_{0}^{y}$, i.e. the $\|\cdot\|_{\mathcal{M}_{n}}$ norm. But, here also, such a choice is impossible in $S_{0}^{x} \times S_{0}^{y}$ but is possible in $S^{x} \times S^{y}$ (with a local estimate in a discrete $\mathrm{H}^{2}$ norm, as we shall see later), building $\boldsymbol{v}$ through a convenient discretization of the Dirichlet problem.

We now describe this choice for a general MAC grid $\mathcal{M}$. Let $\rho=\left\{\rho_{i, j}, i=\right.$ $1, \ldots, N, j=1, \ldots, M\} \in S$ and let $w=\left\{w_{i, j}, i=1, \ldots, N, j=1, \ldots, M\right\} \in S$ be the finite volume solution (on the mesh associated to $p$ and $\rho$ ) of $-\Delta w=\rho$ with an homogeneous Dirichlet boundary condition, that is:

$$
-\Delta_{\mathcal{M}} w=\rho,
$$

where $\Delta_{\mathcal{M}} w \in S$ is defined by, for all $i \in\{1, \ldots, N\}, j \in\{1, \ldots, M\}$,

$$
\left(\Delta_{\mathcal{M}} w\right)_{i, j}=\frac{w_{i+1, j}-w_{i, j}}{h_{i+\frac{1}{2}}^{x} h_{i}^{x}}-\frac{w_{i, j}-w_{i-1, j}}{h_{i-\frac{1}{2}}^{x} h_{i}^{x}}+\frac{w_{i, j+1}-w_{i, j}}{h_{j+\frac{1}{2}}^{y} h_{j}^{y}}-\frac{w_{i, j}-w_{i, j-1}}{h_{j-\frac{1}{2}}^{y} h_{j}^{y}},
$$

with, for all $i \in\{0, \ldots, N+1\}$ and $j \in\{0, \ldots, M+1\}$,

$$
w_{0, j}=w_{N+1, j}=w_{i, 0}=w_{i, M+1}=0 .
$$

Let us then take $\boldsymbol{v}=-\bar{\nabla}_{\mathcal{M}} w$ where $\bar{\nabla}_{\mathcal{M}}$ is a discrete gradient of $w$ defined by $\bar{\nabla}_{\mathcal{M}} w=\left(\left(\bar{\nabla}_{\mathcal{M}} w\right)^{x},\left(\bar{\nabla}_{\mathcal{M}} w\right)^{y}\right)^{t} \in S^{x} \times S^{y}$ with:

$$
\begin{aligned}
& \left(\bar{\nabla}_{\mathcal{M}} w\right)_{i+\frac{1}{2}, j}^{x}=\frac{w_{i+1, j}-w_{i, j}}{h_{i+\frac{1}{2}}^{x}}, i \in\{0, \ldots, N\}, j \in\{1, \ldots, M\}, \\
& \left(\bar{\nabla}_{\mathcal{M}} w\right)_{i, j+\frac{1}{2}}^{y}=\frac{w_{i, j+1}-w_{i, j}}{h_{j+\frac{1}{2}}^{y}}, i \in\{1, \ldots, N\}, j \in\{0, \ldots, M\} .
\end{aligned}
$$


Note that in general $\bar{\nabla}_{\mathcal{M}} w$ does not belong to $S_{0}^{x} \times S_{0}^{y}$. This choice of $\boldsymbol{v}$ (using the finite volume discretization of the Laplace operator on the grid associated to $\rho$ ) ensures that $\operatorname{div}_{\mathcal{M}} \boldsymbol{v}=\rho$. Furthermore, since $\boldsymbol{v}$ is a discrete gradient given by formulae (7.16), it satisfies $\operatorname{curl}_{\mathcal{M}} \boldsymbol{v}=0$ (relation (7.13) is not used for this property). These two properties are stated in the following lemma.

Lemma 7.2. Let $w=\left\{w_{i, j}, i=1, \ldots, N, j=1, \ldots, M\right\} \in S$. Let $\boldsymbol{v}=\left(v^{x}, v^{y}\right)=$ $-\bar{\nabla}_{\mathcal{M}} w \in S^{x} \times S^{y}$ be defined by (7.16) and (7.15). Then, with the discrete curl operator denoted by $\operatorname{curl}_{\mathcal{M}}$ and defined by (7.10), we have $\operatorname{curl}_{\mathcal{M}} \boldsymbol{v}=0$.

Furthermore, if $w$ satisfies (7.13) with $\rho=\left\{\rho_{i, j}, i=1, \ldots, N, j=1, \ldots, M\right\} \in S$, then $\operatorname{div}_{\mathcal{M}} \boldsymbol{v}=\rho$.

Proof. We first prove that $\operatorname{curl}_{\mathcal{M}} \boldsymbol{v}=0$, which is the discrete counterpart of the classical equality $\operatorname{curl}(\nabla w)=0$ for a regular function $w$. Let $i \in\{1, \ldots, N+1\}$ and $j \in\{1, \ldots, M+1\}$. By definition (7.10), one has, on $K_{i-\frac{1}{2}, j-\frac{1}{2}}, \operatorname{curl}_{\mathcal{M}} \boldsymbol{v}=A-B$ with:

$$
A=\frac{v_{i, j-\frac{1}{2}}^{y}-v_{i-1, j-\frac{1}{2}}^{y}}{h_{i-\frac{1}{2}}^{x}} \text { and } B=\frac{v_{i-\frac{1}{2}, j}^{x}-v_{i-\frac{1}{2}, j-1}^{x}}{h_{j-\frac{1}{2}}^{y}} .
$$

Recall that the values at the boundary are given by:

$$
v_{i-\frac{1}{2}, 0}^{x}=0, v_{j-\frac{1}{2}, M+1}^{x}=0, v_{0, j-\frac{1}{2}}^{y}=0, v_{N+1, j-\frac{1}{2}}^{y}=0 .
$$

We now use (7.16) (and (7.15) for the values at the boundary), that is

$$
\begin{aligned}
& -v_{i+\frac{1}{2}, j}^{x}=\frac{w_{i+1, j}-w_{i, j}}{h_{i+\frac{1}{2}}^{x}}, i \in\{0, \ldots, N\}, j \in\{0, \ldots, M+1\}, \\
& -v_{i, j+\frac{1}{2}}^{y}=\frac{w_{i, j+1}-w_{i, j}}{h_{j+\frac{1}{2}}^{y}}, i \in\{0, \ldots, N+1\}, j \in\{0, \ldots, M\},
\end{aligned}
$$

and we obtain:

$$
A=-\frac{w_{i, j}-w_{i, j-1}}{h_{i-\frac{1}{2}}^{x} h_{j-\frac{1}{2}}^{y}}+\frac{w_{i-1, j}-w_{i-1, j-1}}{h_{i-\frac{1}{2}}^{x} h_{j-\frac{1}{2}}^{y}}, B=-\frac{w_{i, j}-w_{i-1, j}}{h_{j-\frac{1}{2}}^{y} h_{i-\frac{1}{2}}^{x}}+\frac{w_{i, j-1}-w_{i-1, j-1}}{h_{j-\frac{1}{2}}^{y} h_{i-\frac{1}{2}}^{x}},
$$

from which one deduces $A=B$ and then $\operatorname{curl}_{\mathcal{M}} \boldsymbol{v}=0$.

We now prove the second part of the lemma. Let $i \in\{1, \ldots, N\}$ and $j \in$ $\{1, \ldots, M\}$. One has, on $K_{i, j}$, following (3.15),

$$
\operatorname{div}_{\mathcal{M}} \boldsymbol{v}=\frac{1}{h_{i}^{x} h_{j}^{y}}\left(h_{j}^{y} v_{i+\frac{1}{2}, j}^{x}-h_{j}^{y} v_{i-\frac{1}{2}, j}^{x}+h_{i}^{x} v_{i, j+\frac{1}{2}}^{y}-h_{j}^{y} v_{i, j-\frac{1}{2}}^{x}\right) .
$$

Using (7.16), we obtain:

$$
\operatorname{div}_{\mathcal{M}} \boldsymbol{v}=-\frac{w_{i+1, j}-w_{i, j}}{h_{i}^{x} h_{i+\frac{1}{2}}^{x}}+\frac{w_{i, j}-w_{i-1, j}}{h_{i}^{x} h_{i-\frac{1}{2}}^{x}}-\frac{w_{i, j+1}-w_{i, j}}{h_{j}^{y} h_{j+\frac{1}{2}}^{y}}+\frac{w_{i, j}-w_{i, j-1}}{h_{j}^{y} h_{j-\frac{1}{2}}^{y}}=\rho_{i, j} .
$$

which concludes the proof of the lemma. $\square$

REMARK 7.3. As for Lemma 7.1, this proof extends to the three-dimensional case.

As in the continuous setting described in Section 7.1, we now prove, in Lemma 7.3 below, that an $\mathrm{L}^{2}(\Omega)$-estimate on $\rho$ gives a discrete- $\mathrm{H}_{l o c}^{2}(\Omega)$ estimate on $w$ and then a 
discrete- $\mathrm{H}_{l o c}^{1}(\Omega)$ estimate on $\boldsymbol{v}$. In order to state these local estimates, we first define an approximation $\varphi_{\mathcal{M}}$ of a function $\varphi \in \mathrm{C}_{c}^{\infty}(\Omega)$ defined by:

$$
\varphi_{i, j}=\varphi\left(x_{i}, y_{j}\right) \text { for all } i \in\{0, \ldots, N+1\}, j \in\{0, \ldots, M+1\} .
$$

and consider for $w \in S$, the gradient of the function $w \varphi_{\mathcal{M}} \in S$ as defined in (7.16), that is $\bar{\nabla}\left(w \varphi_{\mathcal{M}}\right)=\left(\bar{\nabla}\left(w \varphi_{\mathcal{M}}\right)^{x}, \bar{\nabla}\left(w \varphi_{\mathcal{M}}\right)^{y}\right)^{t}$. Note that if $\varphi=1$, one recovers (using $(7.15)$ as the boundary conditions for $w)\left(\bar{\nabla}_{\mathcal{M}}(w \varphi)\right)^{x}=-v^{x}$ and $\left(\bar{\nabla}_{\mathcal{M}}(w \varphi)\right)^{y}=-v^{y}$ where $\boldsymbol{v}=\left(v^{x}, v^{y}\right)$ is defined by (7.16).

Lemma 7.3. Let $\mathcal{M}$ be a $M A C$ grid, let $\rho \in S$ and let $w \in S$ be the finite volume solution of $-\Delta w=\rho$, with an homogeneous Dirichlet boundary condition, i.e. let $w$ be the solution to (7.13)-(7.15). Let $\varphi \in \mathrm{C}_{c}^{\infty}(\Omega)$. Then, there exists $C_{\varphi}$ only depending on $\varphi, \eta$ (which is such that (3.2) holds) and $\Omega$, such that $\left\|\left(\bar{\nabla}_{\mathcal{M}}(w \varphi)\right)^{x}\right\|_{x} \leq C_{\varphi}\|\rho\|_{\mathrm{L}^{2}(\Omega)}$ and $\left\|\left(\bar{\nabla}_{\mathcal{M}}(w \varphi)\right)^{y}\right\|_{y} \leq C_{\varphi}\|\rho\|_{\mathrm{L}^{2}(\Omega)}$.

Proof. Step 1. We first prove that the discrete $\mathrm{H}_{0}^{1}$-norm of $w$ is controlled by the $\mathrm{L}^{2}$-norm of $\rho$. This is a consequence of a now classical discrete Poincaré inequality, which is quite easy to prove with a Cartesian mesh. Indeed, using

$$
w_{i, j}=\sum_{k=1}^{i}\left(w_{k, j}-w_{k-1, j}\right)=\sum_{k=i}^{N}\left(w_{k, j}-w_{k+1, j}\right)
$$

for $i \in\{1, \ldots, N\}$ and $j \in\{1, \ldots, M\}$, the Cauchy-Schwarz inequality yields:

$$
\|w\|_{\mathrm{L}^{2}(\Omega)}^{2} \leq \sum_{i=0}^{N} \sum_{j=1}^{M} \frac{h_{j}^{y}}{h_{i+\frac{1}{2}}^{x}}\left(w_{i+1, j}-w_{i, j}\right)^{2}
$$

and then $\|w\|_{\mathrm{L}^{2}(\Omega)} \leq\|w\|_{\mathcal{M}}$, with:

$$
\|w\|_{\mathcal{M}}^{2}=\sum_{i=0}^{N} \sum_{j=1}^{M} \frac{h_{j}^{y}}{h_{i+\frac{1}{2}}^{x}}\left(w_{i+1, j}-w_{i, j}\right)^{2}+\sum_{i=1}^{N} \sum_{j=0}^{M} \frac{h_{i}^{x}}{h_{j+\frac{1}{2}}^{y}}\left(w_{i, j+1}-w_{i, j}\right)^{2} .
$$

(Note that we again use the notation $\|\cdot\|_{\mathcal{M}}$ for functions of $S$ whereas it was used for functions of $S_{0}^{x} \times S_{0}^{y}$ before, since there is no possible confusion here). Then, since:

$$
\int_{\Omega} w \Delta_{\mathcal{M}} w \mathrm{~d} \boldsymbol{x}=\|w\|_{\mathcal{M}}^{2}=\int_{\Omega} \rho w \mathrm{~d} \boldsymbol{x} \leq\|\rho\|_{\mathrm{L}^{2}(\Omega)}\|w\|_{\mathrm{L}^{2}(\Omega)},
$$

we obtain:

$$
\|w\|_{\mathcal{M}} \leq \operatorname{diam}(\Omega)\|\rho\|_{\mathrm{L}^{2}(\Omega)} .
$$

Step 2. Let $\eta>0$ be such that (3.2) is satisfied. Let $\varphi \in \mathrm{C}_{c}^{\infty}(\Omega)$ and $\varphi_{\mathcal{M}}$ be its discrete approximation defined by (7.17). Thanks to the discrete $\mathrm{H}_{0}^{1}$-estimate (7.18) on $w$, there exists $C_{\varphi}$ only depending on $\varphi, \eta$ and $\Omega$ such that $\left\|\Delta_{\mathcal{M}}\left(w \varphi_{\mathcal{M}}\right)\right\|_{\mathrm{L}^{2}(\Omega)} \leq$ $C_{\varphi}\|\rho\|_{\mathrm{L}^{2}(\Omega)}$. We now deduce an estimate on $\left\|\left(\bar{\nabla}_{\mathcal{M}}(w \varphi)\right)^{x}\right\|_{x}$ and $\left\|\left(\bar{\nabla}_{\mathcal{M}}(w \varphi)\right)^{y}\right\|_{y}$, using a discrete counterpart of the computation which leads to (7.8). In the following we denote for short by $w \varphi_{i, j}$ the quantity $w_{i, j} \varphi_{i, j}$, for $i \in\{0, \ldots, N+1\}$ and $j \in$ $\{0, \ldots, M+1\}$. With this notation, we remark that $\left\|\left(\bar{\nabla}_{\mathcal{M}}(w \varphi)\right)^{x}\right\|_{x}^{2}=A+B$ with:

$$
A=\sum_{i=1}^{N} \sum_{j=1}^{M}\left(\frac{w \varphi_{i+1, j}-w \varphi_{i, j}}{h_{i+\frac{1}{2}}^{x}}-\frac{w \varphi_{i, j}-w \varphi_{i-1, j}}{h_{i-\frac{1}{2}}^{x}}\right)^{2} \frac{h_{j}^{y}}{h_{i}^{x}},
$$


and:

$$
B=\sum_{i=0}^{N} \sum_{j=1}^{M+1}\left(\frac{w \varphi_{i+1, j}-w \varphi_{i, j}}{h_{i+\frac{1}{2}}^{x}}-\frac{w \varphi_{i+1, j-1}-w \varphi_{i, j-1}}{h_{i+\frac{1}{2}}^{x}}\right)^{2} \frac{h_{i+\frac{1}{2}}^{x}}{h_{j-\frac{1}{2}}^{y}} .
$$

Note that $A$ (resp. $B$ ) is a discrete counterpart of the square of the $\mathrm{L}^{2}$-norm of $\partial_{x} \partial_{x}(w \varphi)\left(\right.$ resp. $\left.\partial_{y} \partial_{x}(w \varphi)\right)$. We now perform a double discrete integration by part to transform $B$ :

$$
\begin{aligned}
B & =\sum_{i=0}^{N} \sum_{j=1}^{M}\left(w \varphi_{i+1, j}-w \varphi_{i, j}-w \varphi_{i+1, j-1}+w \varphi_{i, j-1}\right)\left(w \varphi_{i+1, j}-w \varphi_{i, j}\right) \frac{1}{h_{i+\frac{1}{2}}^{x} h_{j-\frac{1}{2}}^{y}} \\
& -\sum_{i=0}^{N} \sum_{j=1}^{M}\left(w \varphi_{i+1, j+1}-w \varphi_{i, j+1}-w \varphi_{i+1, j}+w \varphi_{i, j}\right)\left(w \varphi_{i+1, j}-w \varphi_{i, j}\right) \frac{1}{h_{i+\frac{1}{2}}^{x} h_{j+\frac{1}{2}}^{y}} .
\end{aligned}
$$

This can be rewritten as:

$$
\begin{array}{r}
B=\sum_{i=0}^{N} \sum_{j=1}^{M}\left(\frac{w \varphi_{i+1, j}-w \varphi_{i+1, j-1}}{h_{j-\frac{1}{2}}^{y}}-\frac{w \varphi_{i+1, j+1}-w \varphi_{i+1, j}}{h_{j+\frac{1}{2}}^{y}}\right) \frac{w \varphi_{i+1, j}-w \varphi_{i, j}}{h_{i+\frac{1}{2}}^{x}} \\
-\sum_{i=0}^{N} \sum_{j=1}^{M}\left(\frac{w \varphi_{i, j}-w \varphi_{i, j-1}}{h_{j-\frac{1}{2}}^{y}}-\frac{w \varphi_{i, j+1}-w \varphi_{i, j}}{h_{j+\frac{1}{2}}^{y}}\right) \frac{w \varphi_{i+1, j}-w \varphi_{i, j}}{h_{i+\frac{1}{2}}^{x}} .
\end{array}
$$

Changing the index in the first summation (and noting that $\varphi_{0, j}=\varphi_{N+1, j}=0$ ) yields:

$$
\begin{aligned}
B=\sum_{i=1}^{N} & \sum_{j=1}^{M}\left(\frac{w \varphi_{i, j}-w \varphi_{i, j-1}}{h_{j-\frac{1}{2}}^{y}}-\frac{w \varphi_{i, j+1}-w \varphi_{i, j}}{h_{j+\frac{1}{2}}^{y}}\right) \frac{w \varphi_{i, j}-w \varphi_{i-1, j}}{h_{i-\frac{1}{2}}^{x}} \\
& -\sum_{i=1}^{N} \sum_{j=1}^{M}\left(\frac{w \varphi_{i, j}-w \varphi_{i, j-1}}{h_{j-\frac{1}{2}}^{y}}-\frac{w \varphi_{i, j+1}-w \varphi_{i, j}}{h_{j+\frac{1}{2}}^{y}}\right) \frac{w \varphi_{i+1, j}-w \varphi_{i, j}}{h_{i+\frac{1}{2}}^{x}}
\end{aligned}
$$

which can be rewritten as:

$$
\begin{aligned}
B=\sum_{i=1}^{N} \sum_{j=1}^{M}\left(\frac{w \varphi_{i, j}-w \varphi_{i, j-1}}{h_{j-\frac{1}{2}}^{y}}\right. & \left.-\frac{w \varphi_{i, j+1}-w \varphi_{i, j}}{h_{j+\frac{1}{2}}^{y}}\right) \\
& \left(\frac{w \varphi_{i, j}-w \varphi_{i-1, j}}{h_{i-\frac{1}{2}}^{x}}-\frac{w \varphi_{i+1, j}-w \varphi_{i, j}}{h_{i+\frac{1}{2}}^{x}}\right) .
\end{aligned}
$$

Adding $A$ to this expression of $B$ gives:

$$
\left\|\left(\bar{\nabla}_{\mathcal{M}}(w \varphi)\right)^{x}\right\|_{x}^{2}=\sum_{i=1}^{N} \sum_{j=1}^{M}\left(\frac{w \varphi_{i+1, j}-w \varphi_{i, j}}{h_{i+\frac{1}{2}}^{x}}-\frac{w \varphi_{i, j}-w \varphi_{i-1, j}}{h_{i-\frac{1}{2}}^{x}}\right)\left(\Delta_{\mathcal{M}}\left(w \varphi_{\mathcal{M}}\right)\right)_{i, j} h_{j}^{y} .
$$

Exchanging $x$ and $y, i$ and $j$, a similar computation yields:

$$
\left\|\left(\bar{\nabla}_{\mathcal{M}}(w \varphi)\right)^{y}\right\|_{y}^{2}=\sum_{i=1}^{N} \sum_{j=1}^{M}\left(\frac{w \varphi_{i, j+1}-w \varphi_{i, j}}{h_{j+\frac{1}{2}}^{y}}-\frac{w \varphi_{i, j}-w \varphi_{i, j-1}}{h_{j-\frac{1}{2}}^{y}}\right)\left(\Delta_{\mathcal{M}}\left(w \varphi_{\mathcal{M}}\right)\right)_{i, j} h_{i}^{x} .
$$


Then, we obtain the desired result:

$$
\begin{aligned}
\left\|\left(\bar{\nabla}_{\mathcal{M}}(w \varphi)\right)^{x}\right\|_{x}^{2}+\left\|\left(\bar{\nabla}_{\mathcal{M}}(w \varphi)\right)^{y}\right\|_{y}^{2} & =\sum_{i=1}^{N} \sum_{j=1}^{M}\left(\Delta_{\mathcal{M}}\left(w \varphi_{\mathcal{M}}\right)\right)_{i, j}^{2} h_{i}^{x} h_{j}^{y} \\
& =\left\|\Delta_{\mathcal{M}}\left(w \varphi_{\mathcal{M}}\right)\right\|_{L^{2}(\Omega)}^{2} \leq C_{\varphi}^{2}\|\rho\|_{L^{2}(\Omega)}^{2} .
\end{aligned}
$$

Under the hypotheses of Proposition 6.1, the sequence $\left(\rho_{n}\right)_{n \in \mathbb{N}}$ is bounded in $\mathrm{L}^{2}(\Omega)$. Let $\left(w_{n}\right)_{n \in \mathbb{N}}$ be the corresponding sequence (defined as in Lemma 7.3) and $\boldsymbol{v}_{n}=\left(\boldsymbol{v}_{n}^{x}, \boldsymbol{v}_{n}^{y}\right)$ with $\boldsymbol{v}_{n}^{x}=-\left(\bar{\nabla}_{n}(w \varphi)\right)^{x}, \boldsymbol{v}_{n}^{y}=-\left(\bar{\nabla}_{n}(w \varphi)\right)^{y}$. Thanks to Lemma 7.3, the compactness theorem 3.1 gives, for all $\varphi \in \mathrm{C}_{c}^{\infty}(\Omega)$, that, as $n \rightarrow \infty$ and up to a subsequence, $\left(\bar{\nabla}_{n}(w \varphi)\right)^{x}$ and $\left(\bar{\nabla}_{n}(w \varphi)\right)^{y}$ converge in $\mathrm{L}^{2}(\Omega)$ and that the limits are in $\mathrm{H}_{0}^{1}(\Omega)$. Then, using a diagonal extraction (with a sequence $\left(\varphi_{p}\right)_{p \in \mathbb{N}} \subset \mathrm{C}_{c}^{\infty}(\Omega)$, $\varphi_{p}=1$ on $K_{p}$ and $\left.\operatorname{dist}\left(K_{p}, \Omega^{c}\right) \leq 1 / p\right)$, we obtain that, up to a subsequence, $\boldsymbol{v}_{n} \rightarrow \boldsymbol{v}$ in $\mathrm{L}_{l o c}^{2}(\Omega)^{2}$ and $\boldsymbol{v} \in \mathrm{H}_{l o c}^{1}(\Omega)^{2}$. Furthermore, using Lemma 7.2 (that is the fact that $\operatorname{div}_{\mathcal{M}} \boldsymbol{v}_{n}=\rho_{n}$ and $\operatorname{curl}_{\mathcal{M}} \boldsymbol{v}_{n}=0$ ), it is quite easy to show that $\operatorname{div} \boldsymbol{v}=\rho$ and curl $\boldsymbol{v}=0$ (see Proposition 7.4).

We can now prove that, for all $\varphi \in \mathrm{C}_{c}^{\infty}(\Omega)$ :

$$
\int_{\Omega}\left(p_{n}-\operatorname{div}_{\mathcal{M}} \boldsymbol{u}_{n}\right) \rho_{n} \varphi \mathrm{d} \boldsymbol{x} \rightarrow \int_{\Omega}(p-\operatorname{div} \boldsymbol{u}) \rho \varphi \mathrm{d} \boldsymbol{x} \text { as } n \rightarrow \infty
$$

This is stated in the following result.

Proposition 7.4. Let $\eta>0$ and $\left(\mathcal{M}_{n}\right)_{n \in \mathbb{N}}$ be a sequence of MAC grids satisfying (3.2), with step size $h^{n}$ tending to zero as $n \rightarrow \infty$. Let $p_{n}$ and $\boldsymbol{u}_{n}$ be solution of the discrete momentum equations, that is (4.2a)-(4.2b), associated to $\mathcal{M}_{n}$. Assume that, as $n \rightarrow \infty, p_{n} \rightarrow p$ weakly in $\mathrm{L}^{2}(\Omega)$ and $\boldsymbol{u}_{n} \rightarrow \boldsymbol{u}$ in $\mathrm{L}^{2}(\Omega)^{2}$ with $\boldsymbol{u} \in \mathrm{H}_{0}^{1}(\Omega)^{2}$. Assume also that there exists $C \in \mathbb{R}$ such that $\left\|\left(\boldsymbol{u}_{n}\right)^{x}\right\|_{x} \leq C$ and $\left\|\left(\boldsymbol{u}_{n}\right)^{y}\right\|_{y} \leq C$. For $n \in \mathbb{N}$ let $\rho_{n} \in S_{n}$ and assume that $\rho_{n} \rightarrow \rho$ weakly in $\mathrm{L}^{2}(\Omega)$. Then, for all $\varphi \in \mathrm{C}_{c}^{\infty}(\Omega)$,

$$
\lim _{n \rightarrow \infty} \int_{\Omega}\left(p_{n}-\operatorname{div}_{\mathcal{M}_{n}} \boldsymbol{u}_{n}\right) \rho_{n} \varphi \mathrm{d} \boldsymbol{x}=\int_{\Omega}(p-\operatorname{div} \boldsymbol{u}) \rho \varphi \mathrm{d} \boldsymbol{x}
$$

Proof. Let $\boldsymbol{v}$ be defined by (7.13)-(7.16). Let $\varphi \in \mathrm{C}_{c}^{\infty}(\Omega)$. For a MAC grid $\mathcal{M}$, we define $\varphi_{\mathcal{M}} \in S, \varphi_{\mathcal{M}}^{x} \in S_{0}^{x}$ and $\varphi_{\mathcal{M}}^{y} \in S_{0}^{y}$ by:

$$
\begin{aligned}
& \varphi_{\mathcal{M}}=\varphi_{i, j}=\varphi\left(x_{i}, y_{j}\right) \text { in } K_{i, j}, 0 \leq i \leq N+1,0 \leq j \leq M+1, \\
& \varphi_{\mathcal{M}}^{x}=\varphi_{i+\frac{1}{2}, j}=\varphi\left(x_{i+\frac{1}{2}}, y_{j}\right) \text { in } K_{i+\frac{1}{2}, j}, 0 \leq i \leq N+1,0 \leq j \leq M+1, \\
& \varphi_{\mathcal{M}}^{y}=\varphi_{i, j+\frac{1}{2}}=\varphi\left(x_{i}, y_{j+\frac{1}{2}}\right) \text { in } K_{i, j+\frac{1}{2}}, 0 \leq i \leq N+1,0 \leq j \leq M+1 .
\end{aligned}
$$

For a sequence of grids $\mathcal{M}_{n}$, for short we shall denote $\varphi_{n}=\varphi_{\mathcal{M}_{n}}$. We define $w_{n}$ with (7.13)-(7.15) (with $\mathcal{M}_{n}$ and $\rho_{n}$ instead of $\mathcal{M}$ and $\rho$ ) and $\boldsymbol{v}_{n}$ with (7.16) (with $\mathcal{M}_{n}$ and $w_{n}$ instead of $\mathcal{M}$ and $\left.w\right)$. We set $\hat{\boldsymbol{v}}_{n}=\left(\boldsymbol{v}_{n}^{x} \varphi_{n}^{x}, \boldsymbol{v}_{n}^{y} \varphi_{n}^{y}\right)$. Since $\hat{\boldsymbol{v}}_{n} \in S_{0}^{x} \times S_{0}^{y}$, it is possible to take $\boldsymbol{v}=\hat{\boldsymbol{v}}_{n}$ in (7.12):

$$
\int_{\Omega} \operatorname{div}_{\mathcal{M}} \boldsymbol{u}_{n} \operatorname{div}_{\mathcal{M}} \hat{\boldsymbol{v}}_{n} \mathrm{~d} \boldsymbol{x}+\int_{\Omega} \operatorname{curl}_{\mathcal{M}} \boldsymbol{u}_{n} \operatorname{curl}_{\mathcal{M}} \hat{\boldsymbol{v}}_{n} \mathrm{~d} \boldsymbol{x}-\int_{\Omega} p_{n} \operatorname{div}_{\mathcal{M}} \hat{\boldsymbol{v}}_{n} \mathrm{~d} \boldsymbol{x}=\int_{\Omega} \boldsymbol{f}_{n} \cdot \hat{\boldsymbol{v}}_{n} \mathrm{~d} \boldsymbol{x} .
$$


We now mimick the proof of (7.9). Since $\operatorname{div}_{\mathcal{M}} \boldsymbol{v}_{n}=\rho_{n}$, we first remark that:

$$
\int_{\Omega} \operatorname{div}_{\mathcal{M}} \boldsymbol{u}_{n} \operatorname{div}_{\mathcal{M}} \hat{\boldsymbol{v}}_{n} \mathrm{~d} \boldsymbol{x}=\int_{\Omega}\left(\operatorname{div}_{\mathcal{M}} \boldsymbol{u}_{n}\right) \rho_{n} \varphi \mathrm{d} \boldsymbol{x}+\int_{\Omega}\left(\operatorname{div}_{\mathcal{M}} \boldsymbol{u}_{n}\right) \boldsymbol{v}_{n} \cdot \boldsymbol{\nabla} \varphi \mathrm{d} \boldsymbol{x}+R_{1, n},
$$

where $\lim _{n \rightarrow \infty} R_{1, n}=0$, thanks to the discrete $\mathrm{H}^{1}(\Omega)$-estimate on $\boldsymbol{u}_{n}$ and the $\mathrm{L}_{l o c}^{2}(\Omega)$ estimate on $\boldsymbol{v}_{n}$. Replacing $\operatorname{div}_{\mathcal{M}} \boldsymbol{u}_{n}$ by $p_{n}$, the same computation gives:

$$
\int_{\Omega} p_{n} \operatorname{div}_{\mathcal{M}} \hat{\boldsymbol{v}}_{n} \mathrm{~d} \boldsymbol{x}=\int_{\Omega} p_{n} \rho_{n} \varphi \mathrm{d} \boldsymbol{x}+\int_{\Omega} p_{n} \boldsymbol{v}_{n} \cdot \nabla \varphi \mathrm{d} \boldsymbol{x}+R_{2, n},
$$

where $\lim _{n \rightarrow \infty} R_{2, n}=0$. Similarly, we transform the second term of (7.20):

$$
\begin{aligned}
\int_{\Omega} \operatorname{curl}_{\mathcal{M}} \boldsymbol{u}_{n} \operatorname{curl}_{\mathcal{M}} \hat{\boldsymbol{v}}_{n} \mathrm{~d} \boldsymbol{x}=\int_{\Omega} \operatorname{curl}_{\mathcal{M}} \boldsymbol{u}_{n}\left(\operatorname{curl}_{\mathcal{M}} \boldsymbol{v}_{n}\right) \varphi \mathrm{d} \boldsymbol{x} \\
+\int_{\Omega}\left(\operatorname{curl}_{\mathcal{M}} \boldsymbol{u}_{n}\right)\left(\overline{\boldsymbol{v}}_{n}^{y} \frac{\partial \varphi}{\partial x}-\overline{\boldsymbol{v}}_{n}^{x} \frac{\partial \varphi}{\partial y}\right) \mathrm{d} \boldsymbol{x}+R_{3, n},
\end{aligned}
$$

where $\lim _{n \rightarrow \infty} R_{3, n}=0$ (for the same reasons as $R_{1, n}$ ) and with $\overline{\boldsymbol{v}}_{n}=\left(\overline{\boldsymbol{v}}_{n}^{x}, \overline{\boldsymbol{v}}_{n}^{y}\right.$ ) where $\overline{\boldsymbol{v}}_{n}^{x}$ (and similarly $\overline{\boldsymbol{v}}_{n}^{y}$ ) is defined as follows:

$$
\overline{\boldsymbol{v}}_{n}^{x}=\frac{h_{j+1}^{y}}{2 h_{j+\frac{1}{2}}^{y}}\left(\boldsymbol{v}_{n}^{x}\right)_{i+\frac{1}{2}, j+1}+\frac{h_{j}^{y}}{2 h_{j+\frac{1}{2}}^{y}}\left(\boldsymbol{v}_{n}^{x}\right)_{i+\frac{1}{2}, j} \text { on } K_{i+\frac{1}{2}, j+\frac{1}{2}} .
$$

Since $\operatorname{curl}_{\mathcal{M}} \boldsymbol{v}_{n}=0,(7.23)$ leads to:

$$
\int_{\Omega} \operatorname{curl}_{\mathcal{M}} \boldsymbol{u}_{n} \operatorname{curl}_{\mathcal{M}} \hat{\boldsymbol{v}}_{n} \mathrm{~d} \boldsymbol{x}=\int_{\Omega}\left(\operatorname{curl}_{\mathcal{M}} \boldsymbol{u}_{n}\right) L(\varphi) \overline{\boldsymbol{v}}_{n} \mathrm{~d} \boldsymbol{x}+R_{3, n},
$$

where $L(\varphi)$ is the same matrix involving the first order derivatives of $\varphi$ as in the proof of $(7.9)$.

We recall now that, thanks to Lemma 7.3 , since $\rho_{n}$ is bounded in $\mathrm{L}^{2}(\Omega)$, the compactness theorem 3.1 gives that, up to a subsequence, as $n \rightarrow \infty, \boldsymbol{v}_{n}$ converges to some $\boldsymbol{v}$ in $\mathrm{L}_{l o c}^{2}(\Omega)^{2}$ and that $\boldsymbol{v} \in \mathrm{H}_{l o c}^{1}(\Omega)^{2}$. Thanks to its definition, the $\mathrm{L}_{l o c}^{2}(\Omega)^{2}$ limit of $\overline{\boldsymbol{v}}_{n}$ is the same as $\boldsymbol{v}_{n}$, namely $\boldsymbol{v}$. Indeed, using (3.2) and the discrete- $H_{l o c}^{1}(\Omega)^{2}$ estimate on $\boldsymbol{v}_{n}$, it is quite easy to show that $\left(\boldsymbol{v}_{n}-\overline{\boldsymbol{v}}_{n}\right) \rightarrow 0$ in $\mathrm{L}_{l o c}^{2}(\Omega)^{2}$ as $n \rightarrow \infty$. As a consequence of the compactness theorem 3.1 we also have that $\operatorname{div}_{\mathcal{M}} \boldsymbol{u}_{n}$ and $\operatorname{curl}_{\mathcal{M}} \boldsymbol{u}_{n}$ converge weakly in $L^{2}(\Omega)$ towards $\operatorname{div} \boldsymbol{u}$ and curlu. By hypothesis, we have the weak convergence of $p_{n}$ in $L^{2}(\Omega)$ towards $p$. Then, using (7.24)-(7.21), we deduce from (7.20):

$\lim _{n \rightarrow \infty} \int_{\Omega}\left(\operatorname{div}_{\mathcal{M}} \boldsymbol{u}_{n}-p_{n}\right) \rho_{n} \varphi \mathrm{d} \boldsymbol{x}=\int_{\Omega}((p-\operatorname{div} \boldsymbol{u}) \boldsymbol{v} \cdot \nabla \varphi-(\operatorname{curl} \boldsymbol{u}) L(\varphi) \boldsymbol{v}+\boldsymbol{f} \cdot \boldsymbol{v} \varphi) \mathrm{d} \boldsymbol{x}$.

Finally, since $p_{n}$ and $\boldsymbol{u}_{n}$ are solution of the discrete momentum balance equations, we already know thanks to the estimates on $p_{n}$ and $\rho_{n}$ that the limits $p$ and $\boldsymbol{u}$ are solution of the momentum balance equation; hence, since $\boldsymbol{v} \in \mathrm{H}_{l o c}^{1}(\Omega)^{2}$ :

$$
\begin{aligned}
& \int_{\Omega}(\operatorname{div} \boldsymbol{u}-p)(\operatorname{div} \boldsymbol{v}) \varphi \mathrm{d} \boldsymbol{x}= \\
& \qquad \int_{\Omega}((p-\operatorname{div} \boldsymbol{u}) \boldsymbol{v} \cdot \nabla \varphi-(\operatorname{curl} \boldsymbol{u}) L(\varphi) \boldsymbol{v}+\operatorname{curl} \boldsymbol{u}(\operatorname{curl} \boldsymbol{v}) \varphi+\boldsymbol{f} \cdot \boldsymbol{v} \varphi) \mathrm{d} \boldsymbol{x}
\end{aligned}
$$


But, thanks to the discrete $H_{l o c}^{1}(\Omega)^{2}$-estimates on $\boldsymbol{v}_{n}$, it is quite easy to prove (as in Theorem 3.1) that $\operatorname{div}_{\mathcal{M}} \boldsymbol{v}_{n}$ and $\operatorname{curl}_{\mathcal{M}} \boldsymbol{v}_{n}$ converge weakly in $L_{l o c}^{2}(\Omega)$ towards div $\boldsymbol{v}$ and $\operatorname{curl} \boldsymbol{v}$. This gives that $\operatorname{div} \boldsymbol{v}=\rho$ and $\operatorname{curl} \boldsymbol{v}=0$ and therefore:

$$
\int_{\Omega}(\operatorname{div} \boldsymbol{u}-p) \rho \varphi \mathrm{d} \boldsymbol{x}=\int_{\Omega}((p-\operatorname{div} \boldsymbol{u}) \boldsymbol{v} \cdot \nabla \varphi-(\operatorname{curl} \boldsymbol{u}) L(\varphi) \boldsymbol{v}+\boldsymbol{f} \cdot \boldsymbol{v} \varphi) \mathrm{d} \boldsymbol{x} .
$$

Then, we obtain the desired result, that is:

$$
\lim _{n \rightarrow \infty} \int_{\Omega}\left(p_{n}-\operatorname{div} \boldsymbol{u}_{n}\right) \rho_{n} \varphi \mathrm{d} \boldsymbol{x}=\int_{\Omega}(p-\operatorname{div} \boldsymbol{u}) \rho \varphi \mathrm{d} \boldsymbol{x} .
$$

We now want to replace in (7.25) the function $\varphi$ (which is in $\mathrm{C}_{c}^{\infty}(\Omega)$ ) by the constant function equal to 1 . Let us set $F_{n}=\left(p_{n}-\operatorname{div}_{\mathcal{M}} \boldsymbol{u}_{n}\right) \rho_{n}$. The estimate on $\left(p_{n}-\operatorname{div}_{\mathcal{M}} \boldsymbol{u}_{n}\right)$ in $\mathrm{L}^{2}(\Omega)$ and the estimate on $\rho_{n}$ in $\mathrm{L}^{2 \gamma}(\Omega)$ give, thanks to the Hölder inequality, an estimate on $F_{n}$ in $\mathrm{L}^{q}(\Omega)$ for $q=2 \gamma /(\gamma+1)>1$ (since $\gamma>1$ ). This yields the equi-integrability of the family $\left(F_{n}\right)_{n \in \mathbb{N}}$. Then Lemma B.2 of [5] and (7.25) give the desired result, namely:

$$
\lim _{n \rightarrow \infty} \int_{\Omega}\left(p_{n}-\operatorname{div}_{\mathcal{M}} \boldsymbol{u}_{n}\right) \rho_{n} \mathrm{~d} \boldsymbol{x}=\int_{\Omega}(p-\operatorname{div} \boldsymbol{u}) \rho \mathrm{d} \boldsymbol{x} .
$$

But thanks to the mass equations (discrete and continuous, that is $(2.2 \mathrm{~b})$ and $(4.2 \mathrm{c})$ ), Lemma 2.1 of [5] and Lemma 5.4 give:

$$
\int_{\Omega} \rho_{n} \operatorname{div}_{\mathcal{M}} \boldsymbol{u}_{n} \mathrm{~d} \boldsymbol{x} \leq C h_{n}^{\alpha}, \quad \int_{\Omega} \rho \operatorname{div} \boldsymbol{u} \mathrm{d} \boldsymbol{x}=0 .
$$

Then, we conclude that

$$
\liminf _{n \rightarrow \infty} \int_{\Omega} p_{n} \rho_{n} \mathrm{~d} \boldsymbol{x} \leq \limsup _{n \rightarrow \infty} \int_{\Omega} p_{n} \rho_{n} \mathrm{~d} \boldsymbol{x} \leq \int_{\Omega} p \rho \mathrm{d} \boldsymbol{x} .
$$

7.3. A.e. and strong convergence of $\rho_{n}$ and $p_{n}$. Let us now prove the a.e. convergence of $\rho_{n}$ and $p_{n}$. Let $G_{n}=\left(\rho_{n}^{\gamma}-\rho^{\gamma}\right)\left(\rho_{n}-\rho\right)$. One has $G_{n} \in \mathrm{L}^{1}(\Omega)$ and $G_{n} \geq 0$ a.e. in $\Omega$. Futhermore:

$$
\int_{\Omega} G_{n} \mathrm{~d} \boldsymbol{x}=\int_{\Omega} p_{n} \rho_{n} \mathrm{~d} \boldsymbol{x}-\int_{\Omega} p_{n} \rho \mathrm{d} \boldsymbol{x}-\int_{\Omega} \rho^{\gamma} \rho_{n} \mathrm{~d} \boldsymbol{x}+\int_{\Omega} \rho^{\gamma} \rho \mathrm{d} \boldsymbol{x} .
$$

Using the weak convergence in $\mathrm{L}^{2}(\Omega)$ of $p_{n}$ and $\rho_{n}$, and (7.26), we obtain:

$$
\liminf _{n \rightarrow \infty} \int_{\Omega} G_{n} \mathrm{~d} \boldsymbol{x} \leq 0 .
$$

Then (up to a subsequence), $G_{n} \rightarrow 0$ a.e. and then $\rho_{n} \rightarrow \rho$ a.e. (since $y \mapsto y^{\gamma}$ is an increasing function on $\left.\mathbb{R}_{+}\right)$. Finally, $\rho_{n} \rightarrow \rho$ in $\mathrm{L}^{q}(\Omega)$ for all $1 \leq q<2 \gamma$, $p_{n}=\rho_{n}^{\gamma} \rightarrow \rho^{\gamma}$ in $\mathrm{L}^{q}(\Omega)$ for all $1 \leq q<2$, and $p=\rho^{\gamma}$. We have thus proved the convergence of the approximate pressure and density, which, together with Proposition 6.1 concludes the proof of Theorem 4.1. 
8. Discussion. In this paper, we considered the MAC scheme for the stationary barotropic compressible Stokes equations. This scheme, which is very popular in the computational fluid dynamics community, also proved to be quite adapted to a convergence analysis. Although, for simplicity, we only wrote the MAC scheme for the unit square, the convergence analysis holds for any finite union of rectangles or rectangular parallelepipeds in $3 \mathrm{D}$, as stated in the convergence theorem 4.1.

To our knowledge, this convergence analysis seems to be the first for a finite volume discretization of the compressible Stokes problem. Beside the convergence of the scheme, these analyses also provide an existence result for solutions of the continuous problem (which could also be derived from the continuous existence theory ingredients for the Navier-Stokes equations, as stated in [17, p. 162]). In this respect, we would like to note that the strong convergence of the density and pressure, which is the difficult point of this study, is performed by mimicking a proof for the convergence of perturbations of continuous Stokes equations [5, Theorem 2.2]. A crucial step of this proof is the fact that the integral of the product of the pressure times the density converges, as described in [5, Proof of Theorem 2.2, Step 3] and section 7.1 of the present paper; this way of proving the strong convergence of the pressure results from a (slight) simplification of the original proof [17].

An easy extension of this work consists in replacing the diffusive term $-\Delta \boldsymbol{u}$ in (2.1) by its complete expression $-\mu \Delta \boldsymbol{u}-\mu / 3 \boldsymbol{\nabla}(\operatorname{div} \boldsymbol{u})$ with $\mu>0$ (i.e. the usual form of the divergence of the shear stress tensor in a constant viscosity compressible flow), which is a more realistic term for the model problem (2.1). Then, say for the $x$ component, one replaces the quantity $-\Delta^{x} u^{x}$ in $4.2 \mathrm{a}$ by $-\mu \Delta^{x} u^{x}-\mu / 3 \nabla_{\mathcal{M}}^{x}\left(\operatorname{div}_{\mathcal{M}} \boldsymbol{u}\right)$. Another easy extension is to take gravity effects, that is to replace $\boldsymbol{f}$ by $\boldsymbol{f}+\rho \boldsymbol{g}$, with $\boldsymbol{f} \in \mathrm{L}^{2}(\Omega)^{d}$ and $\boldsymbol{g} \in \mathrm{L}^{\infty}(\Omega)^{d}$. In both cases, the convergence results given above are still true.

Ongoing work concerns the extension to the steady state and transient NavierStokes equations in two or three space dimensions.

Appendix A. Weak formulations of the mass balance equation. For the sake of completeness, we add here a proof of a known result, used in order to prove $(2.2 \mathrm{~b})$. Indeed, the proof that the limit of the approximate solutions satisfies $(2.2 \mathrm{~b})$ is done for a test function $\varphi \in \mathrm{C}_{c}^{\infty}(\Omega)$ rather than for $\varphi \in W^{1, \infty}(\Omega)$. The following lemma (Lemma A.1) proves that this is indeed also true with $\varphi \in W^{1, \infty}(\Omega)$. Lemma A.1 is given with $\rho \in \mathrm{L}^{2}(\Omega)$ and $\boldsymbol{u} \in\left(\mathrm{H}_{0}^{1}(\Omega)\right)^{d}$, which is the case needed for the present paper (and allows a nice proof using the Hardy inequality). Similar results are possible with different assumptions on $\boldsymbol{u}$ and $\rho$ (for instance, $\rho \in \mathrm{L}^{\infty}(\Omega)$ and $\boldsymbol{u} \in\left(W_{0}^{1,1}(\Omega)^{d}\right)$. However, the fact that $\rho \boldsymbol{u} \in \mathrm{L}^{1}(\Omega)$ is obviously not sufficient to ensure that $(2.2 \mathrm{~b})$ is true with $\varphi \in W^{1, \infty}(\Omega)$ if it is true for $\varphi \in \mathrm{C}_{c}^{\infty}(\Omega)$.

Lemma A.1. Let $\Omega$ be a bounded open set of $\mathbb{R}^{d}$, with a Lipschitz continuous boundary. Let $\boldsymbol{u} \in\left(\mathrm{H}_{0}^{1}(\Omega)\right)^{d}$ and $\rho \in \mathrm{L}^{2}(\Omega)$ such that, for all $\varphi \in \mathrm{C}_{c}^{\infty}(\Omega)$,

$$
\int_{\Omega} \rho \boldsymbol{u} \cdot \nabla \varphi \mathrm{d} \boldsymbol{x}=0 .
$$

Then (A.1) holds for all $\varphi \in W^{1, \infty}(\Omega)$.

Proof. Step 1. We first prove that (A.1) holds for all $\varphi \in \mathrm{C}_{c}^{\infty}\left(\mathbb{R}^{d}\right)$. Using the regularity of the boundary, there exists $C \in \mathbb{R}$, only depending on $\Omega$, an increasing 
sequence $\left(K_{n}\right)_{n \in \mathbb{N}} \subset \Omega$ and a sequence $\left(\varphi_{n}\right)_{n \in \mathbb{N}} \subset \mathrm{C}_{c}^{\infty}(\Omega)$ such that:

$$
\begin{aligned}
& \varphi_{n}=1 \text { on } K_{n}, 0 \leq \varphi_{n} \leq 1 \text { on } \Omega, d\left(K_{n}, \mathbb{R}^{d} \backslash \Omega\right) \leq 1 / n, \\
& \left|\nabla \varphi_{n}\right| \leq C n, m\left(C_{n}\right) \leq C / n \text { with } C_{n}=\Omega \backslash K_{n},
\end{aligned}
$$

where $m\left(C_{n}\right)$ denotes the $d$-dimensional Lebesgue measure of $C_{n}$. Let $\varphi \in \mathrm{C}_{c}^{\infty}\left(\mathbb{R}^{d}\right)$.

Since $\varphi \varphi_{n} \in \mathrm{C}_{c}^{\infty}(\Omega)$ for all $n \in \mathbb{N}$, one has $\int_{\Omega} \rho \boldsymbol{u} \cdot \nabla\left(\varphi \varphi_{n}\right) \mathrm{d} \boldsymbol{x}=0$.

This gives that $\int_{\Omega} \rho \boldsymbol{u} \varphi_{n} \cdot \nabla \varphi \mathrm{d} \boldsymbol{x}+\int_{\Omega} \rho \boldsymbol{u} \varphi \cdot \nabla \varphi_{n} \mathrm{~d} \boldsymbol{x}=0$.

The Dominated Convergence Theorem gives that:

$$
\lim _{n \rightarrow \infty} \int_{\Omega} \rho \boldsymbol{u} \varphi_{n} \cdot \nabla \varphi \mathrm{d} \boldsymbol{x}=\int_{\Omega} \rho \boldsymbol{u} \cdot \nabla \varphi \mathrm{d} \boldsymbol{x} .
$$

Then, in order to obtain (A.1), it remains to prove that:

$$
\lim _{n \rightarrow \infty} \int_{\Omega} \rho \boldsymbol{u} \varphi \cdot \nabla \varphi_{n} \mathrm{~d} \boldsymbol{x}=0
$$

This is an easy consequence of the Hardy Inequality, namely of the fact that, thanks to $\boldsymbol{u} \in \mathrm{H}_{0}^{1}(\Omega)^{d}$, one has $\boldsymbol{u} / \delta(\boldsymbol{x}) \in \mathrm{L}^{2}(\Omega)^{d}$, where $\delta(\boldsymbol{x})$ in the distance from $x$ to the boundary of $\Omega$. More precisely, using the Cauchy Schwarz inequality, the assumptions on $\varphi_{n}$ and $\delta(x) n \leq 1$ for all $x \in C_{n}$, one has:

$$
\left|\int_{\Omega} \rho \boldsymbol{u} \varphi \cdot \nabla \varphi_{n} \mathrm{~d} \boldsymbol{x}\right| \leq C_{\varphi} C n \int_{C_{n}}|\rho \boldsymbol{u}| \mathrm{d} \boldsymbol{x} \leq C_{\varphi} C\left[\int_{C_{n}} \rho^{2} \mathrm{~d} \boldsymbol{x}\right]^{\frac{1}{2}}\left[\int_{C_{n}}\left(\frac{\boldsymbol{u}}{\delta}\right)^{2} \mathrm{~d} \boldsymbol{x}\right]^{\frac{1}{2}},
$$

where $C_{\varphi}$ only depends on $\varphi$. The right hand side of this inequality tends to 0 as $n \rightarrow \infty$ since $\rho, \boldsymbol{u} / \delta \in\left(\mathrm{L}^{2}(\Omega)\right)^{d}$ and $\lim _{n \rightarrow \infty} m\left(C_{n}\right)=0$. This gives (A.1) for $\varphi \in \mathrm{C}_{c}^{\infty}\left(\mathbb{R}^{d}\right)$.

Step 2. Proof of (A.1) for $\varphi \in W^{1, \infty}(\Omega)$.

Let $\varphi \in W^{1, \infty}(\Omega)$. The function $\varphi$ is the restriction to $\Omega$ of an element of $W^{1, \infty}\left(\mathbb{R}^{d}\right)$, still denoted by $\varphi$. Let $\left(r_{n}\right)_{n \in \mathbb{N}^{\star}}$ be a sequence of mollifiers, that is:

$$
\begin{aligned}
r \in \mathrm{C}_{c}^{\infty}\left(\mathbb{R}^{d}, \mathbb{R}\right), \int_{\mathbb{R}^{d}} r \mathrm{~d} \boldsymbol{x}=1, r \geq 0 \text { in } \mathbb{R}^{d} & \text { and, for } n \in \mathbb{N}^{\star}, \boldsymbol{x} \in \mathbb{R}^{d}, r_{n}(\boldsymbol{x})=n^{d} r(n \boldsymbol{x}) .
\end{aligned}
$$

Setting $\varphi_{n}=\varphi \star r_{n}$, one has, for all $n \in \mathbb{N}^{\star}, \int_{\Omega} \rho \boldsymbol{u} \cdot \nabla \varphi_{n} \mathrm{~d} \boldsymbol{x}=0$.

Since $\nabla \varphi_{n}=(\nabla \varphi) \star r_{n}$, using the Dominated Convergence Theorem, one gets (A.1).

प

Remark A.1. The hypothesis $\rho \in \mathrm{L}^{2}(\Omega)$ is sharp in Lemma A.1, as we show now by a counterexample. Let $q<2$ (with $q \geq 6 / 5$ if $d=3$ and $q>1$ if $d=2$ ). Let us construct $\rho$ and $\boldsymbol{u}$ such that (A.1) does not imply (2.2b).

Take $\rho \in \mathrm{L}^{q}(\Omega)$ and $\boldsymbol{u} \in\left(\mathrm{H}_{0}^{1}(\Omega)\right)^{d}$ (note that $\left.\rho \boldsymbol{u} \in \mathrm{L}^{1}(\Omega)^{d}\right)$. Let us assume that $\Omega=(0,2) \times(-1,1)^{d-1}$. Let $\alpha \in\left(\frac{1}{2}, \frac{1}{q}\right)$ and let $\rho$ and $\boldsymbol{u}=\left(u_{1}, \ldots, u_{d}\right)^{t}$ be defined as 
follows:

$$
\begin{aligned}
& u_{1}(\boldsymbol{x})=\left\{\begin{array}{l}
x_{1}^{\alpha} \prod_{i=2}^{d}\left(1-\left|x_{i}\right|\right) \text { if } \boldsymbol{x} \in \Omega, x_{1} \leq 1, \\
\left(2-x_{1}\right)^{\alpha} \prod_{i=2}^{d}\left(1-\left|x_{i}\right|\right) \text { if } x \in \Omega, x_{1}>1,
\end{array}\right. \\
& u_{2}=\ldots=u_{d}=0, \\
& \rho(\boldsymbol{x})=\left\{\begin{array}{l}
\frac{1}{x_{1}^{\alpha}} \text { if } x \in \Omega, x_{1} \leq 1, \\
\frac{1}{\left(2-x_{1}\right)^{\alpha}} \text { if } x \in \Omega, x_{1}>1 .
\end{array}\right.
\end{aligned}
$$

We have $\rho \in \mathrm{L}^{q}(\Omega)$ (thanks to $\alpha q<1$ ) and $\boldsymbol{u} \in\left(\mathrm{H}_{0}^{1}(\Omega)\right)^{d}$ (thanks to $2 \alpha>1$ ). Since $\rho u_{1}$ does not depends on $x_{1}$, if is easy to see (integrating by parts) that (A.1) holds. Taking now $\varphi \in \mathrm{C}_{c}^{\infty}\left(\mathbb{R}^{d}\right)$ with, for instance $\varphi=0$ outside $(-1,1) \times(-1 / 2,1 / 2)^{d-1}$, one has:

$$
\int_{\Omega} \rho \boldsymbol{u} \cdot \nabla \varphi \mathrm{d} \boldsymbol{x}=-\int_{-\frac{1}{2}, \frac{1}{2}\left[{ }^{d-1}\right.} \prod_{i=2}^{d}\left(1-\left|x_{i}\right|\right) \varphi(0, y) d y
$$

where $y=\left(x_{2}, \ldots, x_{d}\right)$. It is possible to choose $\varphi$ such that $\varphi(0, y)>0$ for all $y \in(-1 / 2,1 / 2)^{d-1}$. This gives:

$$
\int_{\Omega} \rho \boldsymbol{u} \cdot \nabla \varphi \mathrm{d} \boldsymbol{x}<0
$$

and proves that $(2.2 \mathrm{~b})$ is not true.

Appendix A. Discrete Laplace operators and $\mathrm{H}^{1}$ norms. The discrete Laplace operator $\Delta^{x}$ is such that, $\forall v^{x} \in S_{0}^{x}, \Delta^{x} v^{x} \in S_{0}^{x}$ and:

for $2 \leq i \leq N$, for $2 \leq j \leq M-1$,

$$
\begin{aligned}
h_{i-\frac{1}{2}}^{x} h_{j}^{y}\left(-\Delta^{x} v^{x}\right)_{i-\frac{1}{2}, j} & =\frac{h_{j}^{y}}{h_{i}^{x}}\left(v_{i-\frac{1}{2}, j}^{x}-v_{i+\frac{1}{2}, j}^{x}\right)+\frac{h_{i-\frac{1}{2}}^{x}}{h_{j+\frac{1}{2}}^{y}}\left(v_{i-\frac{1}{2}, j}^{x}-v_{i-\frac{1}{2}, j+1}^{x}\right) \\
+ & \frac{h_{j}^{y}}{h_{i-1}^{x}}\left(v_{i-\frac{1}{2}, j}^{x}-v_{i-\frac{3}{2}, j}^{x}\right)+\frac{h_{i-\frac{1}{2}}^{x}}{h_{j-\frac{1}{2}}^{y}}\left(v_{i-\frac{1}{2}, j}^{x}-v_{i-\frac{1}{2}, j-1}^{x}\right),
\end{aligned}
$$

for $2 \leq i \leq N$

$$
\begin{array}{r}
h_{i-\frac{1}{2}}^{x} h_{1}^{y}\left(-\Delta^{x} v^{x}\right)_{i-\frac{1}{2}, 1}=\frac{h_{1}^{y}}{h_{i}^{x}}\left(v_{i-\frac{1}{2}, 1}^{x}-v_{i+\frac{1}{2}, 1}^{x}\right)+\frac{h_{i-\frac{1}{2}}^{x}}{h_{\frac{3}{2}}^{y}}\left(v_{i-\frac{1}{2}, 1}^{x}-v_{i-\frac{1}{2}, 2}^{x}\right) \\
\quad+\frac{h_{1}^{y}}{h_{i-1}^{x}}\left(v_{i-\frac{1}{2}, 1}^{x}-v_{i-\frac{3}{2}, 1}^{x}\right)+2 \frac{h_{i-\frac{1}{2}}^{x}}{h_{1}^{y}}\left(v_{i-\frac{1}{2}, 1}^{x}\right), \\
h_{i-\frac{1}{2}}^{x} h_{M}^{y}\left(-\Delta^{x} v^{x}\right)_{i-\frac{1}{2}, M}=\frac{h_{M}^{y}}{h_{i}^{x}}\left(v_{i-\frac{1}{2}, M}^{x}-v_{i+\frac{1}{2}, M}^{x}\right)+2 \frac{h_{i-\frac{1}{2}}^{x}}{h_{M}^{y}}\left(v_{i-\frac{1}{2}, M}^{x}\right) \\
+\frac{h_{M}^{y}}{h_{i-1}^{x}}\left(v_{i-\frac{1}{2}, M}^{x}-v_{i-\frac{3}{2}, M}^{x}\right)+\frac{h_{i-\frac{1}{2}}^{x}}{h_{M-\frac{1}{2}}^{y}}\left(v_{i-\frac{1}{2}, M}^{x}-v_{i-\frac{1}{2}, M-1}^{x}\right) .
\end{array}
$$


The operator $\Delta^{y}$ is such that, $\forall v^{y} \in S^{y}, \Delta^{y} v^{y} \in S^{y}$ and:

for $2 \leq i \leq N-1$, for $2 \leq j \leq M$,

$$
\begin{aligned}
h_{i}^{x} h_{j-\frac{1}{2}}^{y}\left(-\Delta^{y} v^{y}\right)_{i, j-\frac{1}{2}} & =\frac{h_{j-\frac{1}{2}}^{\bar{y}}}{h_{i+\frac{1}{2}}^{x}}\left(v_{i, j-\frac{1}{2}}^{y}-v_{i+1, j-\frac{1}{2}}^{y}\right)+\frac{h_{i}^{x}}{h_{j}^{y}}\left(v_{i, j-\frac{1}{2}}^{y}-v_{i, j+\frac{1}{2}}^{y}\right) \\
+ & \frac{h_{j-\frac{1}{2}}^{y}}{h_{i-\frac{1}{2}}^{x}}\left(v_{i, j-\frac{1}{2}}^{y}-v_{i-1, j-\frac{1}{2}}^{y}\right)+\frac{h_{i}^{x}}{h_{j-1}^{y}}\left(v_{i, j-\frac{1}{2}}^{y}-v_{i, j-\frac{3}{2}}^{y}\right),
\end{aligned}
$$

for $2 \leq j \leq M$

$$
\begin{aligned}
& h_{1}^{x} h_{j-\frac{1}{2}}^{y}\left(-\Delta^{y} v^{x}\right)_{1, j-\frac{1}{2}}=\frac{h_{j-\frac{1}{2}}^{y}}{h_{\frac{3}{2}}^{x}}\left(v_{1, j-\frac{1}{2}}^{y}-v_{2, j-\frac{1}{2}}^{y}\right)+\frac{h_{1}^{x}}{h_{j}^{y}}\left(v_{1, j-\frac{1}{2}}^{y}-v_{1, j+\frac{1}{2}}^{y}\right) \\
& +2 \frac{h_{j-\frac{1}{2}}^{y}}{h_{1}^{x}}\left(v_{1, j-\frac{1}{2}}^{y}\right)+\frac{h_{1}^{x}}{h_{j-1}^{y}}\left(v_{1, j-\frac{1}{2}}^{y}-v_{1, j-\frac{3}{2}}^{y}\right), \\
& h_{N}^{x} h_{j-\frac{1}{2}}^{y}\left(-\Delta^{y} v^{x}\right)_{N, j-\frac{1}{2}}=2 \frac{h_{j-\frac{1}{2}}^{y}}{h_{N}^{x}}\left(v_{N, j-\frac{1}{2}}^{y}\right)+\frac{h_{N}^{x}}{h_{j}^{y}}\left(v_{N, j-\frac{1}{2}}^{y}-v_{N, j+\frac{1}{2}}^{y}\right) \\
& +\frac{h_{j-\frac{1}{2}}^{y}}{h_{N-\frac{1}{2}}^{x}}\left(v_{N, j-\frac{1}{2}}^{y}-v_{N-1, j-\frac{1}{2}}^{y}\right)+\frac{h_{N}^{x}}{h_{j-1}^{y}}\left(v_{N, j-\frac{1}{2}}^{y}-v_{N, j-\frac{3}{2}}^{y}\right) .
\end{aligned}
$$

Using Equations (A.1) and (A.2) and reordering the summations in Equations (3.5), we obtain for the discrete $\mathrm{H}_{0}^{1}$ inner products the following expression:

$$
\begin{aligned}
\forall v^{x} \in S_{0}^{x}, \forall w^{x} \in S_{0}^{x}, & \\
\left(v^{x}, w^{x}\right)^{x}= & \sum_{\substack{1 \leq i \leq N, 1 \leq j \leq M}} \frac{h_{j}^{y}}{h_{i}^{x}}\left(v_{i+\frac{1}{2}, j}^{x}-v_{i-\frac{1}{2}, j}^{x}\right)\left(w_{i+\frac{1}{2}, j}^{x}-w_{i-\frac{1}{2}, j}^{x}\right) \\
+ & \sum_{\substack{2 \leq i \leq N, 1 \leq j \leq M-1}} \frac{h_{i-\frac{1}{2}}^{x}}{h_{j+\frac{1}{2}}^{y}}\left(v_{i-\frac{1}{2}, j+1}^{x}-v_{i-\frac{1}{2}, j}^{x}\right)\left(w_{i-\frac{1}{2}, j+1}^{x}-w_{i-\frac{1}{2}, j}^{x}\right) \\
+ & \sum_{2 \leq i \leq N} 2 \frac{h_{i-\frac{1}{2}}^{x}}{h_{1}^{y}} v_{i-\frac{1}{2}, 1}^{x} w_{i-\frac{1}{2}, 1}^{x}+\sum_{2 \leq i \leq N} 2 \frac{h_{i-\frac{1}{2}}^{x}}{h_{M}^{y}} v_{i-\frac{1}{2}, M}^{x} w_{i-\frac{1}{2}, M}^{x} .
\end{aligned}
$$

$\forall v^{y} \in S_{0}^{y}, \forall w^{y} \in S_{0}^{y}$,

$$
\begin{aligned}
\left(v^{y}, w^{y}\right)^{y}= & =\sum_{\substack{1 \leq i \leq N-1, 2 \leq j \leq M}} \frac{h_{j-\frac{1}{2}}^{y}}{h_{i+\frac{1}{2}}^{x}}\left(v_{i+1, j-\frac{1}{2}}^{y}-v_{i, j-\frac{1}{2}}^{y}\right)\left(w_{i+1, j-\frac{1}{2}}^{y}-w_{i, j-\frac{1}{2}}^{y}\right) \\
& +\sum_{\substack{1 \leq i \leq N, 1 \leq j \leq M}} \frac{h_{i}^{x}}{h_{j}^{y}}\left(v_{i, j+\frac{1}{2}}^{y}-v_{i, j-\frac{1}{2}}^{y}\right)\left(w_{i, j+\frac{1}{2}}^{y}-w_{i, j-\frac{1}{2}}^{y}\right) \\
& +\sum_{2 \leq i \leq M} 2 \frac{h_{j-\frac{1}{2}}^{y}}{h_{1}^{x}} v_{1, j-\frac{1}{2}}^{y} w_{1, j-\frac{1}{2}}^{y}+\sum_{2 \leq i \leq M} 2 \frac{h_{j-\frac{1}{2}}^{y}}{h_{N}^{x}} v_{N, j-\frac{1}{2}}^{y} w_{N, j-\frac{1}{2}}^{y}
\end{aligned}
$$




\section{REFERENCES}

[1] H. Bijl and P. Wesseling. A unified method for computing incompressible and compressible flows in boundary-fitted coordinates. Journal of Computational Physics, 141:153-173, 1998.

[2] V. Casulli and D. Greenspan. Pressure method for the numerical solution of transient, compressible fluid flows. International Journal for Numerical Methods in Fluids, 4:1001-1012, 1984.

[3] P. Colella and K. Pao. A projection method for low speed flows. Journal of Computational Physics, 149:245-269, 1999.

[4] R. Eymard, T. Gallouët, and R. Herbin. Finite volume methods. In P. Ciarlet and J. Lions, editors, Handbook of Numerical Analysis, Volume VII, pages 713-1020. North Holland, 2000.

[5] R. Eymard, T. Gallouët, R. Herbin, and J.-C. Latché. A convergent finite element-finite volume scheme for the compressible Stokes problem. Part II: the isentropic case. Mathematics of Computation, 79:649-675, 2010.

[6] E. Feireisl. Dynamics of viscous compressible flows. volume 26 of Oxford Lecture Series in Mathematics and its Applications. Oxford University Press, 2004.

[7] T. Gallouët, R. Herbin, and J.-C. Latché. A convergent finite element-finite volume scheme for the compressible Stokes problem. Part I: the isothermal case. Mathematics of Computation, 267:1333-1352, 2009.

[8] L. Gastaldo, R. Herbin, and J.-C. Latché. A discretization of phase mass balance in fractional step algorithms for the drift-flux model. to appear in IMA Journal of Numerical Analysis, 2009.

[9] F. Harlow and A. Amsden. Numerical calculation of almost incompressible flow. Journal of Computational Physics, 3:80-93, 1968.

[10] F. Harlow and A. Amsden. A numerical fluid dynamics calculation method for all flow speeds. Journal of Computational Physics, 8:197-213, 1971.

[11] F. Harlow and J. Welsh. Numerical calculation of time-dependent viscous incompressible flow of fluid with free surface. Physics of Fluids, 8:2182-2189, 1965.

[12] R. Issa. Solution of the implicitly discretised fluid flow equations by operator splitting. Journal of Computational Physics, 62:40-65, 1985.

[13] R. Issa, A. Gosman, and A. Watkins. The computation of compressible and incompressible recirculating flows by a non-iterative implicit scheme. Journal of Computational Physics, 62:66-82, 1986.

[14] K. Karki and S. Patankar. Pressure based calculation procedure for viscous flows at all speeds in arbitrary configurations. AIA A Journal, 27:1167-1174, 1989.

[15] K. Karlsen and T. Karper. A convergent mixed method for the Stokes approximation of viscous compressible flow. Preprint, 2009.

[16] K. Karlsen and T. Karper. A convergent nonconforming finite element method for compressible Stokes flow. Preprint, 2009.

[17] P.-L. Lions. Mathematical topics in fluid mechanics - volume 2 - compressible models. volume 10 of Oxford Lecture Series in Mathematics and its Applications. Oxford University Press, 1998.

[18] R. A. Nicolaides. Analysis and convergence of the MAC scheme. I. The linear problem. SIAM Journal on Numerical Analysis, 29:1579-1591, 1992.

[19] R. A. Nicolaides and X. Wu. Analysis and convergence of the MAC scheme. II. Navier-Stokes equations. Mathematics of Computation, 65:29-44, 1996.

[20] A. Novotný and I. Straškraba. Introduction to the mathematical theory of compressible flow. volume 27 of Oxford Lecture Series in Mathematics and its Applications. Oxford University Press, 2004.

[21] D. Shin and J. C. Strikwerda. Inf-sup conditions for finite-difference approximations of the Stokes equations. Australian Mathematical Society. Journal. Series B. Applied Mathematics, 39:121-134, 1997.

[22] D. van der Heul, C. Vuik, and P. Wesseling. Stability analysis of segregated solution methods for compressible flow. Applied Numerical Mathematics, 38:257-274, 2001.

[23] D. van der Heul, C. Vuik, and P. Wesseling. A conservative pressure-correction method for flow at all speeds. Computer \& Fluids, 32:1113-1132, 2003.

[24] D. Vidović, A. Segal, and P. Wesseling. A superlinearly convergent Mach-uniform finite volume method for the Euler equations on staggered unstructured grids. Journal of Computational 
Physics, 217:277-294, 2006.

[25] C. Wall, C. Pierce, and P. Moin. A semi-implicit method for resolution of acoustic waves in low Mach number flows. Journal of Computational Physics, 181:545-563, 2002.

[26] I. Wenneker, A. Segal, and P. Wesseling. A Mach-uniform unstructured staggered grid method. International Journal for Numerical Methods in Fluids, 40:1209-1235, 2002.

[27] P. Wesseling. Principles of computational fluid dynamics. volume 29 of Springer Series in Computational Mathematics. Springer, 2001. 